\title{
A multispectral analysis of the northeastern shell of IC 443
}

\author{
Alexandre Alarie, ${ }^{1,2,3}$ Laurent Drissen, ${ }^{2,3}$ \\ ${ }^{1}$ Instituto de Astronomía, Universidad National Autónoma de México, Apdo. Postal 70264, 04510 Mexico D.F., Mexico \\ ${ }^{2}$ Département de physique, de génie physique et d'optique, Université Laval, Québec, QC, G1V 0A6, Canada \\ ${ }^{3}$ Centre de Recherche en Astrophysique du Québec
}

9 August 2019

\begin{abstract}
We have carried out optical observations of the north-eastern part of the supernova remnant IC 443 using the CFHT imaging spectrograph SITELLE. The observations consist of three multispectral cubes covering an $11^{\prime} \times 11^{\prime}$ area allowing the investigation of both the spatial and spectral variation of 9 emission lines : [O II] $\lambda \lambda 3726+3729,\left[\mathrm{O}_{\mathrm{III}}\right] \lambda \lambda 4959,5007, \mathrm{H} \beta$, $\mathrm{H} \alpha,[\mathrm{N}$ II] $\lambda \lambda 6548,6583$ and $[\mathrm{S}$ II] $\lambda \lambda 6716,6731$. Extinction measurement from the $\mathrm{H} \alpha / \mathrm{H} \beta$ shows significant variation across the observed region with $\mathrm{E}(\mathrm{B}-\mathrm{V})=0.8-1.1$. Electron density measurements using $[\mathrm{S} \mathrm{II}]$ lines indicate densities ranging from 100 up to $2500 \mathrm{~cm}^{-3}$. Models computed with the shock modelling code MAPPINGS are presented and compared with the observations. A combination of complete shock model and truncated ones are required in order to explain the observed spectrum. The shock velocities found in IC 443 are between 20 and $150 \mathrm{~km} \mathrm{~s}^{-1}$ with $75 \mathrm{~km} \mathrm{~s}^{-1}$ being the most prominent velocity. The pre-shock number density varies from 20 to $60 \mathrm{~cm}^{-3}$. A single set of abundances close to solar values combined with varying shock parameters (shock velocity, pre-shock density and shock age) are sufficient to explain to great variation of lines intensities observed in IC 443. Despite the relatively modest spectral resolution of the data $(\mathrm{R} \sim 1500$ at $\mathrm{H} \alpha)$, we clearly separate the red and blue velocity components of the expanding nebula, which show significant morphological differences.
\end{abstract}

Key words: techniques: imaging spectroscopy - proper motions - ISM: individual objects: IC443 - ISM: supernova remnants

\section{INTRODUCTION}

IC $443(\mathrm{G} 189.1+3.0)$ is an evolved galactic supernova remnant (SNR) located in the direction of the galactic anti-center at a distance between 1.5 and $2 \mathrm{kpc}$ (Petre et al. 1988; Welsh \& Sallmen 2003; Ambrocio-Cruz et al. 2017) and an age estimated at around 3000-30,000 years (Petre et al. 1988; Olbert et al. 2001). Because of its large scale $\left(\approx 0.5^{\circ}\right)$ and clear view without any obstruction in the direction of the remnant, IC 443 is known to be an excellent laboratory to study the complex interaction between a SNR and the interstellar and circumstellar medium.

IC 443 has been classified as a mixed-morphology remnant: shell-like and bright structure in radio continuum and in the optical (Lee et al. 2008) and centrally filled in X-ray. Braun \& Strom (1986) suggested that the remnant has evolved inside a preexisting wind-blown bubble, which likely was formed by the remnant's massive progenitor star. This proposed explanation was supported by Troja et al. $(2006,2008)$ based on the analysis of X-ray observations obtained with XMM-Newton. IC 443 is apparently interacting with both atomic and molecular gas (Snell et al. 2005). In the northeastern part, the SNR shock is encountering mostly an atomic medium, and only the atomic lines expected in the post-shock recombining gas are detected (Fesen \& Kirshner 1980). The southern boundary, on the other hand, shows various molecular lines with broad wings suggesting that the shock is propagating into mostly molecular medium (Burton et al. 1988; Dickman et al. 1992; van Dishoeck et al. 1993).

In recent years, there has been considerable interest in studying the complex interaction between the IC 443 SNR with its surrounding molecular clouds at radio and infrared wavelengths (Hezareh et al. 2013; Fang et al. 2013; Su et al. 2014) as well as the determination of the abundances of heavy elements in some part of the remnant from X-ray observations (Troja et al. 2008; Bocchino et al. 2009; Swartz et al. 2015).

Detections of high and very high energy gamma rays have also been reported in the vicinity of IC 443 , which overlap with the object's structure seen in the radio, IR, optical and X-ray bands. First with the MAGIC telescope (Albert et al. 2007), and then early in the Fermi-LAT mission (Abdo et al. 2010; Ackermann et al. 2013). Is is assumed that these emissions are associated with locally accelerated cosmic rays.

Despite all these observations made in recent years, optical spectroscopy, which samples very different physical phenomena and chemical el- ements, are limited to long-slit spectroscopy (Parker 1964; Esipov et al. 1972; Fesen \& Kirshner 1980). 
This present work was undertaken to improve the spectroscopic data available for the north-eastern part of IC 443 where optical lines are present. Multi-spectral cubes obtained with the imaging Fourier transform spectrometer SITELLE are presented and analyzed alongside with shock models to infer shock properties. The paper is organized as follows: in section 2 we present the observations and data reduction; in section 3 we discuss the shock modelling technique and make direct comparisons with observations. A kinematical analysis of the data is presented in section 4 . We conclude this paper with remarks in section 5 .

\section{OBSERVATIONS AND DATA REDUCTION}

\subsection{Observations}

The data presented here consist of three multispectral data cubes obtained with the optical imaging Fourier Transform Spectrometer (iFTS), SITELLE, attached to the 3.6-meter Canada-FranceHawaii Telescope (Drissen et al. 2019). SITELLE delivers multispectral cubes covering a $11^{\prime} \times 11^{\prime}$ field-of-view, sampled with $0.32^{\prime \prime}$ pixels, covering specific bandpasses of the visible band using filters . A single region of IC 443 , centered at RA $6 \mathrm{~h} 17 \mathrm{~m} 36.25 \mathrm{~s}$ and $\mathrm{DEC}+22^{\circ} 49^{\prime} 15^{\prime \prime}$, was observed during the nights of 2016 March 4 , $6 \& 7$ using three filters targeting bright emission lines : $\mathrm{H} \alpha, \mathrm{H} \beta$, [O II] $\lambda 3726+3729$, [O III] $\lambda 4959,5007$, [N II] $\lambda 6548,6583$ and [S II] $\lambda 6716,6731$. SITELLE's spectral resolution $\mathrm{R}$ is adjustable to the need of the observer; we selected relatively modest values of $\mathrm{R}$ (see Table 1) allowing us to clearly separate the emission lines from their neighbors as our primary goal was not to study the kinematics. However, as we show in section 4, the high signal-to-noise reached in the $\mathrm{SN} 3$ filter (around $\mathrm{H} \alpha$ ) allowed us to perform a preliminary kinematical analysis of the target.

\subsection{Data reduction and line fitting}

The data were reduced using SITELLE's dedicated data reduction software ORBS (Martin et al. 2015). Standard reduction procedures were applied (flat, bias, dark) and the flux calibration was performed using a datacube of the spectrophotometric standard GD71 for each filter to define the transmission function at each wavelength. The spectral calibration was done using a helium-neon laser datacube acquired during daytime. A detailed description regarding the reduction process is provided by Martin \& Drissen (2017).

An individual spectrum is shown in Figure 1. It has to be noted that the apparent wiggles on each side of the emission lines are not due to noise but rather to the cardinal sine function (sinc) typical of all Fourier transform spectrometers, as we chose not to apodize the signal. This peculiar instrument line shape is taken into account by the dedicated line-fitting software ORCS (Martin et al. 2016) which was specifically created to deal with SITELLE data and therefore uses sinc-shaped line profiles to accurately fit each emission line. In order to improve the signal to noise ratio, the line flux extraction was performed using a $3 \times 3$ pixel binning, except for the kinematical analysis, where no binning was done. This manipulation reduced the spatial resolution to approximately $1^{\prime \prime} /$ pixel.

\subsection{Flux and line ratio maps}

Using the flux and error maps generated by ORCS, we have created ratio maps and only kept pixels for which the ratios error is smaller than $10 \%$. Flux maps in all emission lines are shown the Appendix
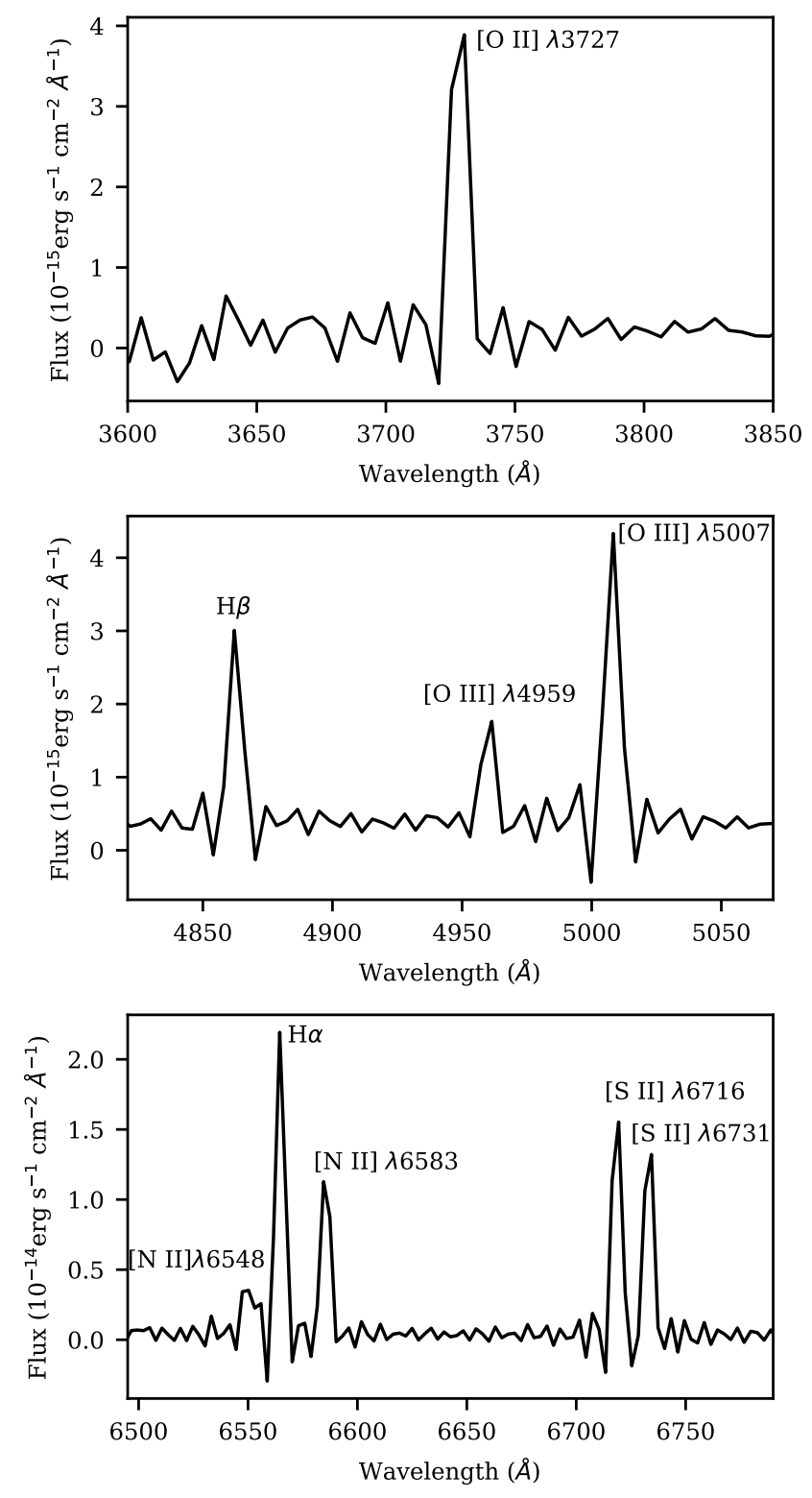

Figure 1. Spectra extracted from the data cubes using a circular aperture $\left(2.22^{\prime \prime}\right)$ centered at RA $6 \mathrm{~h} 17 \mathrm{~m} 57 \mathrm{~s}$ and DEC $22^{\circ} 44^{\prime} 42^{\prime \prime}$.

A : [O II] A1, [O III] A2, $\mathrm{H} \alpha$ A3, [N II] A4 and [S II] A5. An unbinned color composite image of the field, using the $\mathrm{H} \alpha$, [S II] and [O III] flux maps, is shown in Figure 2.

The followings ratios also generated and presented in Appendix A : [O II $] / \mathrm{H} \alpha(\mathrm{A} 7),[\mathrm{O} \mathrm{III}] / \mathrm{H} \beta$ (A8), [O III]/[O II] (A9),

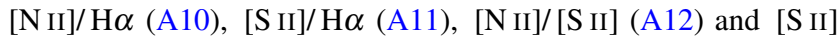
$\lambda 6731 / \lambda 6716$ (A13). Due to insufficient exposure time during the acquisition of the SN1 cube, [O II] emissions could not be accurately measured at many locations leading to incomplete $[\mathrm{O} \mathrm{II}] / \mathrm{H} \alpha$ and $[\mathrm{O} \mathrm{III}] /[\mathrm{O} \mathrm{II}]$ maps.

\subsection{Extinction correction}

IC 443 is well known to contain patchy obscuration over the whole remnant and its surroundings. Since the observations cover an ap- 
Table 1. Characteristics of the multispectral cubes

\begin{tabular}{cccc}
\hline \hline Characteristics & SN1 & SN2 & SN3 \\
\hline Spectral range $(\AA)$ & $3650-3850$ & $4800-5200$ & $6510-6850$ \\
Spectral resolution $(\mathrm{R})$ & 500 & 800 & 1500 \\
Exposure time per image $(\mathrm{s})$ & 30 & 30 & 20 \\
Number of images & 88 & 180 & 266 \\
Total observing time & $44 \mathrm{~min}$ & $1 \mathrm{~h} 30 \mathrm{~m}$ & $1 \mathrm{~h} 28 \mathrm{~m} 40 \mathrm{~s}$ \\
\hline
\end{tabular}

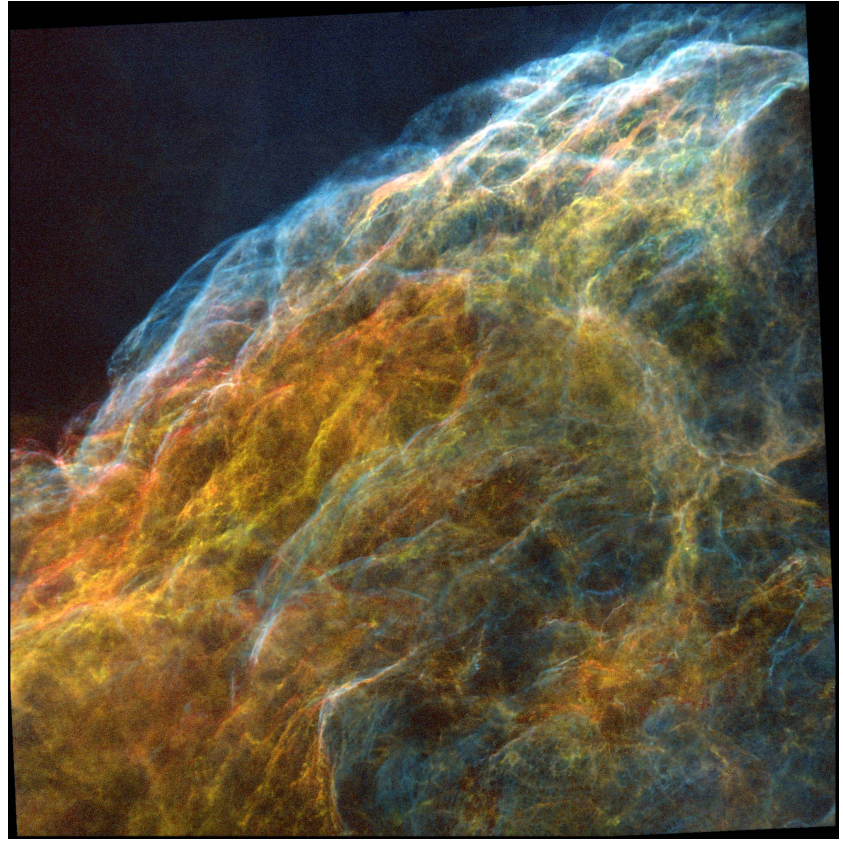

Figure 2. Composite image of IC 443, using SITELLE's SN2 and SN3 data cubes. $\mathrm{H} \alpha$ is coded in red, [S II] $\lambda \lambda 6716,31$ in green and [O III] $\lambda 5007$ in blue. The field of view is $11^{\prime} \times 11^{\prime}$, with East to the left and North at the top.

preciable fraction of the northeastern shell, it is essential to measure the interstellar reddening at various locations and correct the observed line intensities accordingly. For interstellar radiative shocks, the Balmer lines of hydrogen are thought to be primarily produced by recombination with only a small amount of direct collisional excitation from ground level to bound excited states. Shock models calculated by Raymond (1979) have shown that the $\mathrm{H} \alpha / \mathrm{H} \beta$ ratio fall between 2.9 and 3.3 with larger values occurring for the slower shocks. Since we do not have the information about the shock velocity prior to a reduction, we used the standard value of $\mathrm{H} \alpha / \mathrm{H} \beta$ of 3.0 to correct our observations. With this value, we estimated the colour excess $\mathrm{E}(\mathrm{B}-\mathrm{V})$ for every pixel where the error on the $\mathrm{H} \alpha / \mathrm{H} \beta$ ratio is smaller than $10 \%$. The extinction was evaluated using PYNEB (Luridiana et al. 2012) and the REDCORR class which makes use of the Fitzpatrick (1999) correction curve. Since the extinction is most likely diffuse in front of the remnant, we used a Gaussian blur with a standard deviation of $6 \sigma$ in order to smooth the extinction map and fill regions where the extinction could not be directly measured. The map used to correct the line intensities pixel by pixel is shown in Figure A6 ${ }^{1}$. The values found are in agreement with earlier measurements, such as those reported by Fesen \& Kirshner (1980): $\mathrm{E}(\mathrm{B}-\mathrm{V})=0.8-1.1$.

\subsection{Faint nebulosities from the HII region S249}

Some faint nebulosities visible at the northern edge of our field are due to the H II region S249. These emissions undoubtedly contaminate some of IC 443 spectra overlapping these regions. We estimate that the contamination from the brightest $\mathrm{H} \alpha$ and [O III] emission from S249 could contribute up to $10 \%$ and $8 \%$ respectively of the measured emission from IC 443. Despite this contamination, we do not attempt the subtract it from the emissions coming from IC 443. Our decision is based on the fact that both the intensity and shape of the emissions coming from S249 behind IC 443 are unpredictable. It has to be noted that the contamination does not seem to affect the whole observed region, but is rather localized in the northern part of IC 443.

\section{LINE RATIO ANALYSIS}

\subsection{Comparison with previous observations}

Very few spectroscopic observations in the optical regime can be found in the literature. The earliest measurements are presented by Parker (1964) where multiple spectra were taken on the surface of IC 443. Table 2 of their paper shows the line intensities as well as a few line ratios which could be compared with our observations. One position noted as filament $7^{\prime}$ is located in the north-eastern part of the nebula. The exact location of that filament is unknown since its coordinates were not provided. By approximating its location from their finding charts, we compared visually their [N II]/ [S II], $[\mathrm{S} I I] / \mathrm{H} \alpha,[\mathrm{N} \mathrm{II}] / \mathrm{H} \alpha$ and $[\mathrm{O} \mathrm{III}] / \mathrm{H} \beta$ ratios with our ratio maps and found a very similar set of values for multiple locations around the same area.

So far, the most extensive spectroscopic analysis in the optical was carried out by Fesen \& Kirshner (1980). They targetted six positions in IC 443, among which two (their numbers 1 and 4) are located inside our field of view. The positions were sufficiently well indicated to allow a good comparison. As mentioned earlier, their observed $\mathrm{H} \alpha / \mathrm{H} \beta$ ratio is very similar to ours, which lead to the same measurement of the extinction. Also, the line ratios at those places such as $[\mathrm{O} \mathrm{III}] / \mathrm{H} \alpha,[\mathrm{O}$ II $] / \mathrm{H} \alpha,[\mathrm{N} \mathrm{II}] / \mathrm{H} \alpha,[\mathrm{S} \mathrm{II}] / \mathrm{H} \alpha$ and [S II] $\lambda 6716 / \lambda 6731$ are in very good agreement with our measurements. Furthermore, Fesen \& Kirshner (1980) compared their observations with shock models to investigate both the shock conditions and elemental abundances.

\footnotetext{
1 The map can be found in the supplementary material in FITS format.
} 
The data presented in this paper can also be compared with shock models in order to investigate shock conditions at the surface of IC 443. Using SITELLE's data with both the spectral and spatial informations, we can attempt to associate spatial line intensity variations directly with shock conditions. The next section is dedicated to the analysis of shock models combined with our observations. Further comparisons are made between the analysis of Fesen \& Kirshner (1980) and previous modelling of shock-wave spectra.

\subsection{Comparison with new shock models}

Several shock models have been published (Shull \& McKee 1979; Raymond 1979; Dopita \& Sutherland 1996; Allen et al. 2008) and proved useful to study the properties of a great variety of astrophysical objects, especially evolved supernova remnants such as the Cygnus Loop and IC 443. Despite their undeniable usefulness, each grid only covers a very limited set of shock parameters and limit the analysis that can be done for a specific object. To circumvent this problem, we built our own shock models in an attempt to reconcile physical condition found in IC 443 and emission line variations derived from our observations.

We used the shock modelling code MAPPINGS V (Sutherland \& Dopita 2017). This is an updated version of previous releases: MAPPINGS IV (Dopita et al. 2013), MAPPINGS III (Groves et al. 2004a,b; Allen et al. 2008), MAPPINGS II (Sutherland \& Dopita 1993) and MAPPINGS I (Binette et al. 1982; Dopita et al. 1982; Binette et al. 1985) which were themselves based on an earlier version of the code created by Michael A. Dopita (Dopita 1976, 1977; Dopita et al. 1977; Dopita 1978, 1979; Dopita et al. 1980). Details concerning the intricate physics and computational recipes inside the code can be found among the cited papers.

Modelling shock wave spectra require the knowledge of several shock parameters used as input during computation. In the next sections, each parameter is explored independently in order to simplify the overall analysis and discussion. Comparisons with published shock models are made as well as comparisons between the conditions derived from previous analysis on IC 443 and those derived from our observations.

\subsubsection{Preionization}

The ionization state of the precursor gas is a strong function of shock velocity and its value greatly affects the detailed structure of the shock, hence the prediction of the emissivities as mentioned by Raymond (1979). Since this is not a parameter that can be determined directly from our observations, we assume that the shocks present in IC 443 are fast enough to ionise the gas in front of them and no external source other than shocks exists. The computation of the pre-ionization was calculated self-consistently by MAPPINGS V as described in (Sutherland \& Dopita 2017). The pre-ionization was evaluated only once using solar abundance for shock between 50 and $200 \mathrm{~km} \mathrm{~s}^{-1}$ with pre-shock density of $20 \mathrm{~cm}^{-3}$ and a transverse magnetic field of $1 \mu \mathrm{G}$. The result is shown in Fig.3. Since the pre-ionization changes very little under similar shock parameters, we have re-used the same pre-ionization values function of the shock velocity for all models computed in this paper. Preventing the evaluation of the pre-ionization for each model allows faster computation, therefore resulting in virtually the same results concerning emissivity predictions. The models presented in this paper do not include the emission emanating from the precursor gas since

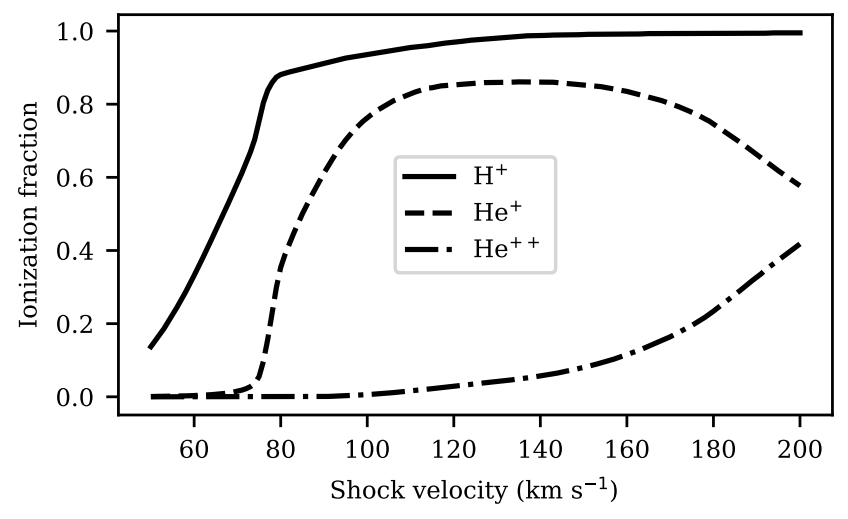

Figure 3. Ionization fraction of $\mathrm{H}^{+}, \mathrm{He}^{+}$and $\mathrm{He}^{++}$versus the shock velocity used as pre-ionization for all shock models computed and shown in this paper.

the shock velocities are not fast enough $\left(<150 \mathrm{~km} \mathrm{~s}^{-1}\right)$ to produce any appreciable emission, as mentioned in Dopita \& Sutherland (1996).

\subsubsection{Line intensity evolution}

Fast shocks are effective at ionizing the gas; the faster the shock, the larger the volume of gas is ionized and the longer it will take to recombine. The recombination time depends on several parameters such as the shock velocity, the pre-shock density and the abundances. Whatever the set of parameters, the highest ionization species recombine first, followed by the lowest. This means that the emission lines intensities vary with time. The rate at which the recombination occurs depends on many complex processes and is determined by shock modelling codes such as MAPPINGS. As an example, Fig. 4 shows the emission line intensity variation for [O III] $\lambda 5007$, [O II] $\lambda \lambda 3727+3729$, [N II] $\lambda 6584$, [S II] $\lambda \lambda 6716+6731$ and $\mathrm{H} \alpha$ for a $100 \mathrm{~km} \mathrm{~s}^{-1}$ shock propagating into a region with an electron density of $20 \mathrm{~cm}^{-3}$. It can be seen that [O III] recombines before $\mathrm{H} \alpha$ and at a certain time both emission are superimposed. This time delay thus leads to a variation of the $\left[\mathrm{O}_{\mathrm{III}}\right] / \mathrm{H} \alpha$ ratio with time. A high $[\mathrm{O} \mathrm{III}] / \mathrm{H} \beta$ is thus a good indicator of a recent shock and can be used as a compass showing the direction of the shock front. Fig. A8 clearly shows this phenomenon, where the $[\mathrm{O} \mathrm{III}] / \mathrm{H} \beta$ ratio indicates the regions where shocks are the most recent and at the same time indicates the direction of the blast wave. The same happens with the $[\mathrm{O} \mathrm{II}] / \mathrm{H} \alpha$ ratio (Fig. A7). This effect is far less obvious in our observations with the [N II]/ $\mathrm{H} \alpha$ and [S II]/H $\alpha$ ratios since the physical separation between [N II], [S II] and $\mathrm{H} \alpha$ are not as large as those for [O III] and [O II].

It must be mentioned that the emission line structure generated behind a shock can barely be observed from our observations considering their spatial resolution. At the distance of IC 443, estimated to be $1.9 \mathrm{kpc}$ (Ambrocio-Cruz et al. 2017), our seeinglimited spatial resolution of $1^{\prime \prime}$ corresponds to a spatial distance of $2.8 \times 10^{14} \mathrm{~cm}$. With the example given in Fig. 4 and for similar shock parameters, nearly all of the observed emission lines are located within a single pixel in our intensity and ratios maps. Therefore, we suppose that the emission coming from one pixel can be interpreted with the help of one shock model in which the total emissivities can be summed.

The parameter used in this paper to compute truncated mod- 
Table 2. Solar abundances with respect to hydrogen used during model calculation based on the compilation of Asplund et al. 2009, Scott et al. 2014 and Grevesse et al. 2014. The abundance of nitrogen and sulfur was modified according to our observations and explained in section 3.2.5.

\begin{tabular}{|c|c|c|}
\hline Atom \# & Abundances & $\log (\mathrm{X} / \mathrm{H})$ \\
\hline 1 & Hydrogen & 0 \\
\hline 2 & Helium & -1.07 \\
\hline 3 & Lithium & -10.95 \\
\hline 4 & Beryllium & -10.62 \\
\hline 5 & Boron & -9.30 \\
\hline 6 & Carbon & -3.57 \\
\hline 7 & Nitrogen & -3.95 \\
\hline 8 & Oxygen & -3.31 \\
\hline 9 & Fluorine & -7.44 \\
\hline 10 & Neon & -4.07 \\
\hline 11 & Sodium & -5.79 \\
\hline 12 & Magnesium & -4.41 \\
\hline 13 & Aluminum & -5.57 \\
\hline 14 & Silicon & -4.49 \\
\hline 15 & Phosphorus & -6.59 \\
\hline 16 & Sulfur & -4.50 \\
\hline 17 & Chlorine & -6.50 \\
\hline 18 & Argon & -5.60 \\
\hline 19 & Potassium & -6.96 \\
\hline 20 & Calcium & -5.68 \\
\hline 21 & Scandium & -8.84 \\
\hline 22 & Titanium & -7.10 \\
\hline 23 & Vanadium & -8.11 \\
\hline 24 & Chromium & -6.38 \\
\hline 25 & Manganese & -6.58 \\
\hline 26 & Iron & -4.53 \\
\hline 27 & Cobalt & -7.07 \\
\hline 28 & Nickel & -5.80 \\
\hline 29 & Copper & -7.82 \\
\hline 30 & Zinc & -7.44 \\
\hline
\end{tabular}

els of incomplete shocks is the cut-off temperature $\left(\mathrm{T}_{c u t}\right)$. It corresponds to the temperature of a region located a certain distance behind the shock at which the computation of a model has been stopped. A model can be considered complete when $\mathrm{T}_{\text {cut }}$ reaches $1000 \mathrm{~K}$ below which no further significant emission in the considered species is produced. Very few truncated models can be found in the literature and were computed to match very precise object such as filaments in the Cygnus Loop (Raymond et al. 1980; Contini \& Shaviv 1982; Raymond et al. 1988) and a low-excitation Herbig-Haro object (Binette et al. 1985).

The large $[\mathrm{O} \mathrm{III}] / \mathrm{H} \beta$ ratios found in some regions of IC 443 suggest the presence of incomplete shocks and the absence of grids with truncated models re-enforced the need to compute one specially adapted to IC 443, taking in consideration the other shock parameters covered in the next sections.

\subsubsection{Shock velocity}

When only observations in the optical regime are available, the shock velocity can derived from the [O III] $\lambda 5007$ to [O II] $\lambda 3727$ ratio, as shown by Dopita (1977) (Fig. 7). His figure can be slightly modified to take into account incomplete shock models. Fig. 4 shows that the $[\mathrm{O} \mathrm{III}]$ and $[\mathrm{O} \mathrm{II}]$ emissivities vary differently with time, therefore influencing the $[\mathrm{O} \mathrm{III}] /[\mathrm{O} \mathrm{II}]$ ratio at a given moment. Fig. 5 shows the variation of the $[\mathrm{O} \mathrm{III}] /[\mathrm{O} \mathrm{II}]$ ratio as a

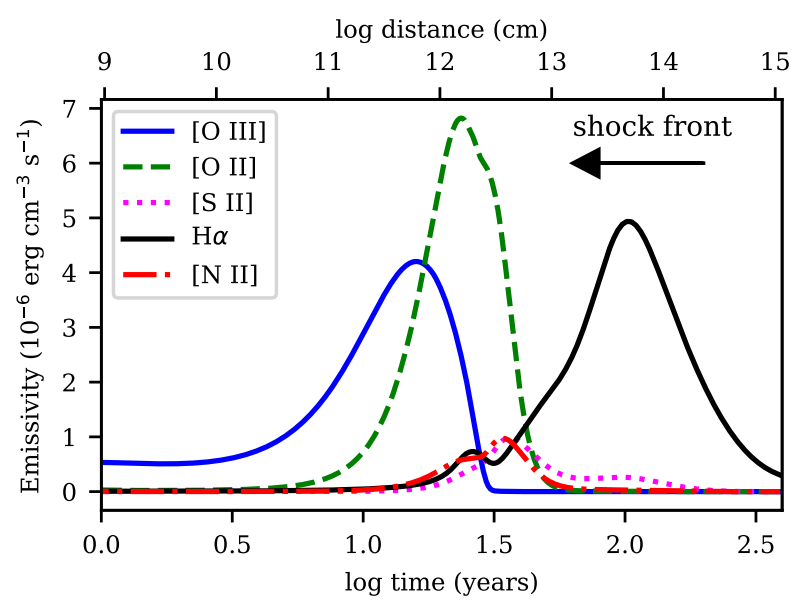

Figure 4. Emissivity profile of [O III] $\lambda 5007$, [O II] $\lambda \lambda 3726+3729$, [N II] $\lambda 6583$, [S II] $\lambda \lambda 6716+6731$ and $\mathrm{H} \alpha$ versus the distance (bottom) and the distance (top) for a $100 \mathrm{~km} \mathrm{~s}^{-1}$ shock propagating into a density of $\mathrm{n}_{0}=20$ $\mathrm{cm}^{-3}$ and a magnetic field of $\mathrm{B}_{0}=1 \mu \mathrm{G}$. The abundances used are given Table 2 .

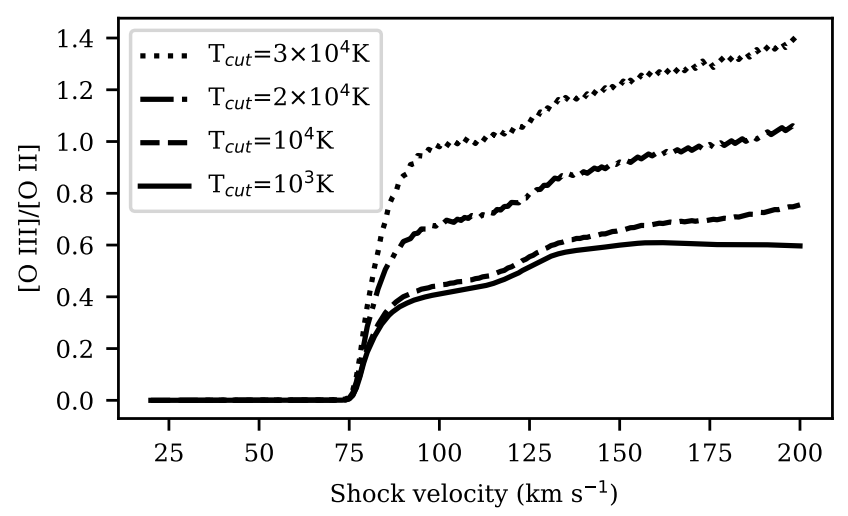

Figure 5. Variation of the $[\mathrm{O} \mathrm{III}] \lambda 5007 /[\mathrm{O}$ II $] \lambda \lambda 3726+3729$ ratio as a function of the shock velocity and cut-off temperatures for $\mathrm{n}_{0}=20 \mathrm{~cm}^{-3}$ and $\mathrm{B}_{0}=1 \mu \mathrm{G}$. The abundances used are given Table 2 .

function of the shock velocity and fixed cut-off temperature during model evaluation. Two important points can be made from this figure:

- The $[\mathrm{O} \mathrm{III}] /[\mathrm{O} \mathrm{II}]$ ratio becomes useful at a shock velocity around $75 \mathrm{~km} \mathrm{~s}^{-1}$, coinciding with the emergence of [O III] $\lambda \lambda 4959,5007$ emission. It has to be noted that the velocity at which [O III] appears depend on the ionization state of the precursor gas during model evaluation. For example, a shock propagating into a neutral medium requires at least a $100 \mathrm{~km} \mathrm{~s}^{-1}$ shock to produce [O III]. In either case, fast shock can be identified directly by observing filaments emitting [O III], as seen in Fig. A2. Regions without [O III], while emitting lower ionization lines such as $\mathrm{H} \alpha$, $[\mathrm{N} \mathrm{II}]$ and $[\mathrm{S} \mathrm{II}]$, indicate the presence of slower shocks $(<75 \mathrm{~km}$ $\mathrm{s}^{-1}$ ).

- Deriving a shock velocity with the [O III]/[O II] ratio requires a precise knowledge of the shock age. Even then, when the shock can be considered as complete $\left(\mathrm{T}_{\text {cut }}=10^{3} \mathrm{~K}\right)$, the usefulness of the $[\mathrm{O} \mathrm{III}] /[\mathrm{O} \mathrm{II}]$ ratio is limited to a shock velocity range between 75 


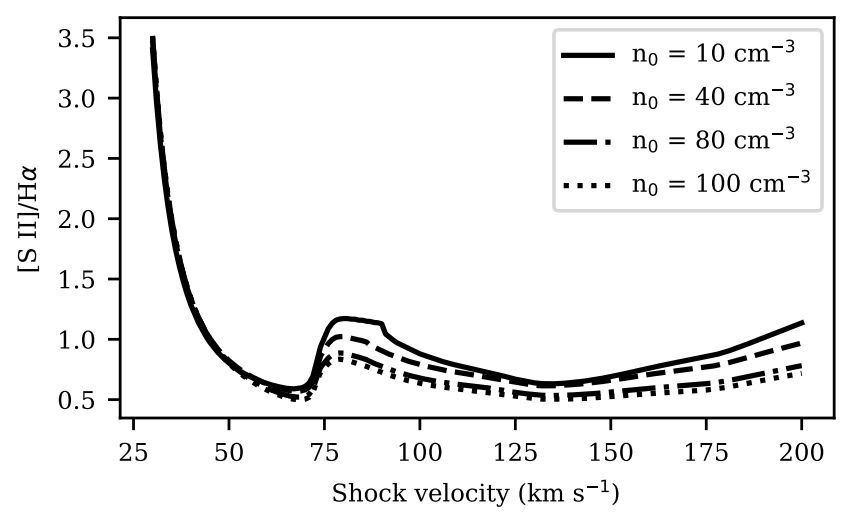

Figure 6. Variation of the $\left[\mathrm{S}_{\mathrm{II}}\right] \lambda \lambda 6716+6731 / \mathrm{H} \alpha$ ratio as a function of the shock velocity and pre-shock densities for $\mathrm{B}_{0}=1 \mu \mathrm{G}$. The abundances used are given Table 2.

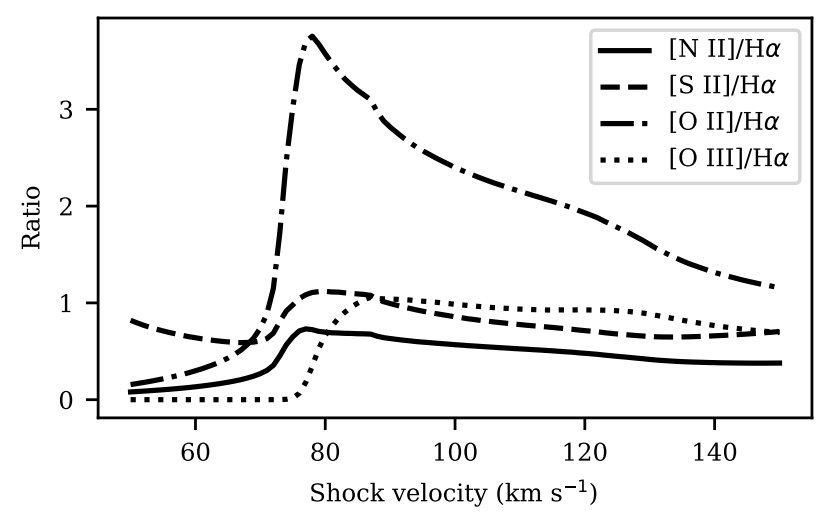

Figure 7. Variation of the $\left[\mathrm{O} \mathrm{III}_{\mathrm{II}}\right] / \mathrm{H} \alpha, \quad\left[\mathrm{O}_{\mathrm{II}}\right] / \mathrm{H} \alpha, \quad[\mathrm{N} \mathrm{II}] / \mathrm{H} \alpha$ and $[\mathrm{S} \mathrm{II}] / \mathrm{H} \alpha$ ratios for shock velocities between 50 and $150 \mathrm{~km} \mathrm{~s}^{-1}, \mathrm{n}_{0}=$ $20 \mathrm{~cm}^{-3}$ and $\mathrm{B}_{0}=1 \mu \mathrm{G}$. The abundances used are given Table 2 .

and $200 \mathrm{~km} \mathrm{~s}^{-1}$, faster shocks producing [O IV] at the expense of [O III]. Despite these limitations, the $[\mathrm{O}$ III]/ [O II] ratio can still be useful to approximate element abundances and will be discussed in section 3.2.5.

As mentioned above, the absence of [O III], along with the presence of lower ionization lines is a good indication of regions being excited by slower shocks. Another indicator that this is the case is the presence of a particularly high $[\mathrm{S}$ II $] / \mathrm{H} \alpha$ ratio, as seen in Fig. A11. Shock models presented in Fig. 6 indicate that shocks with a velocity lower than $30 \mathrm{~km} \mathrm{~s}^{-1}$ could be responsible for the observed emissions and does not depend on the pre-shock density.

The $[\mathrm{N} \mathrm{II}] / \mathrm{H} \alpha$ ratio is also linked to the shock velocity as shown in Fig 7 alongside with other ratios. We can see from Fig. A10 that the $[\mathrm{N} I I] / \mathrm{H} \alpha$ ratios are lower in locations where the $[\mathrm{S} \mathrm{II}] / \mathrm{H} \alpha$ are higher and [O III] absent, therefore, where the shock velocities are the slowest.

\subsubsection{Pre-shock density}

Electron densities can be found using the [S II] $\lambda 6716 / \lambda 6731$ (Weedman 1968) which are available from our data. Measurements obtained by others, using $[\mathrm{O} \mathrm{II}]$ and $[\mathrm{S} \mathrm{II}]$ lines, show clear vari-

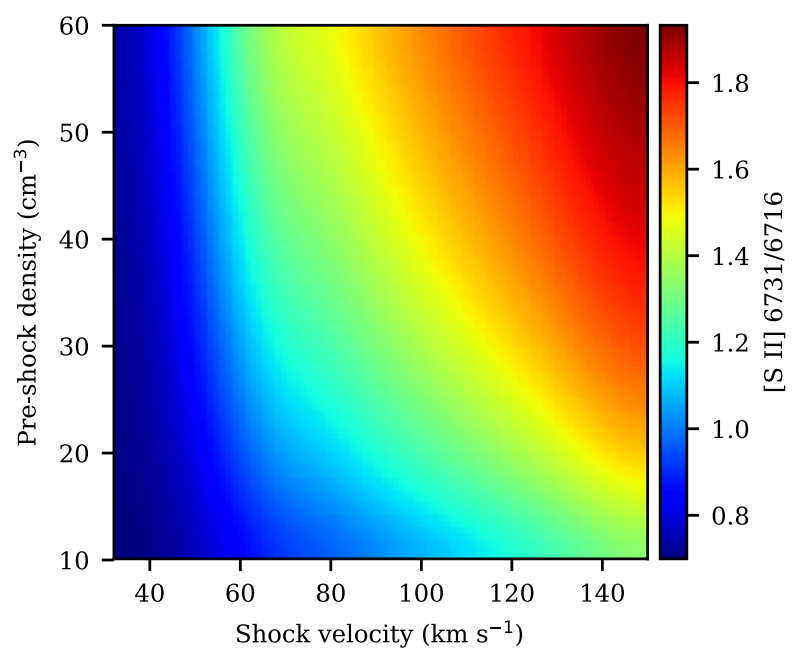

Figure 8. Variation of the $[\mathrm{S}$ II] $\lambda 6731 / \lambda 6716$ ratio as a function of the preshock density and the shock velocity for solar abundances and $\mathrm{B}_{0}=1$ $\mu \mathrm{G}$. This figure was made using 16384 individual shock models evaluated with MAPPINGS.

ations across the nebula. Measurements of brights filaments (Osterbrock 1958) and three positions by Parker (1964) show density fluctuations within the range of $100-600 \mathrm{~cm}^{-3}$ with an average of $350 \mathrm{~cm}^{-3}$. Fesen \& Kirshner (1980) found slightly different values, with electron densities ranging from 100 to $400 \mathrm{~cm}^{-3}$, with an average of $200 \mathrm{~cm}^{-3}$. Using infrared observations, Rho et al. (2001) found an electronic density of $500 \mathrm{~cm}^{-3}$.

Figure A13 shows the variation of the $[\mathrm{S} \mathrm{II}]$ ratio from our observations. The ratio varies from 0.70 to 1.30 , with an average of 1.22. Using PYNEB (Luridiana et al. 2012, 2015) with the atomic data of sulphur taken from the CHIANTI database version 8 (Dere et al. 1997; Del Zanna et al. 2015), we calculated the corresponding electronic densities, which translate a minimum and maximum being respectively 106 and $2477 \mathrm{~cm}^{-3}$. These values are in agreements between previous measurements, but ours show regions with significantly higher electronic densities. Most of them are located in the northeastern part of IC 443, but also in some localised filaments as well. This indicates that the blast wave is encountering a higher pre-shock density in those regions.

Values of the pre-shock density can be estimated with the help of Fig. 8. Assuming a constant magnetic field of $1 \mu \mathrm{G}$ throughout IC 443 , the pre-shock density can be estimated when the shock velocity is known. Using the [S II] $\lambda 6716 / \lambda 6731$ ratio, and [O III]/ [O II] ratio to determine the shock velocity, we found that most pre-shock densities vary between 20 and $60 \mathrm{~cm}^{-3}$. These values are higher than those measured by Fesen \& Kirshner (1980), but this is not surprising since we measured higher electronic densities. We could not measure lower pre-shock densities since the velocity could not be measured at all locations. Measuring the preshock density for slow shocks $\left(<40 \mathrm{~km} \mathrm{~s}^{-1}\right)$ could prove challenging since the $[\mathrm{S}$ II] $\lambda 6731 / \lambda 6716$ ratio then varies very little, as shown in Fig. 8. This effect can be seen by comparing the locations of high $[\mathrm{S} \mathrm{II}] / \mathrm{H} \alpha$ ratio (slow-shocks-Fig. A11) and the [S II] ratio map (Fig. A13), where no clear relation can be seen between $[\mathrm{S} \mathrm{II}] / \mathrm{H} \alpha$ line and $[\mathrm{S} \mathrm{II}] \lambda 6731 / \lambda 6716$ line ratios morphologies. 

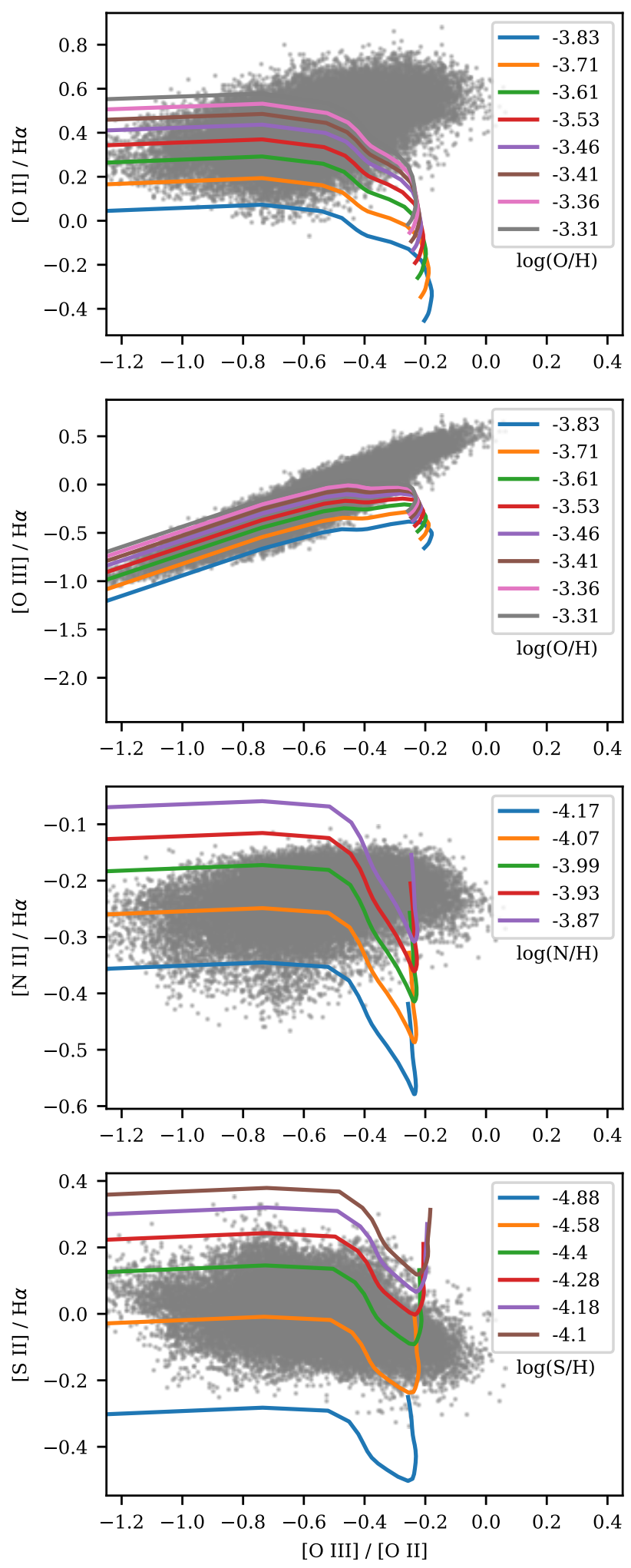

Figure 9. Diagnostic diagram of $[\mathrm{O} \mathrm{II}] / \mathrm{H} \alpha,[\mathrm{O} \mathrm{III}] / \mathrm{H} \alpha,[\mathrm{N} \mathrm{II}] / \mathrm{H} \alpha$ and $[\mathrm{S} \mathrm{II}] / \mathrm{H} \alpha$ versus $[\mathrm{O} \mathrm{III}] /[\mathrm{O}$ II $]$ ratio. The point cloud represent the observations and the colored lines shock models evaluated with different metallicities for shock velocity between $70 \mathrm{~km} \mathrm{~s}^{-1}$ and $200 \mathrm{~km} \mathrm{~s}^{-1}, \mathrm{n}_{0}=20 \mathrm{~cm}^{-3}$ and $\mathrm{B}_{0}=1 \mu \mathrm{G}$.

\subsubsection{Abundance range estimates of $O, N$ and $S$}

With the emissions of [O III], [O II], [N II], [S II] and $\mathrm{H} \alpha$, it is possible to estimate the probable abundances of oxygen, nitrogen and sulphur. In order to achieve this, it is required to estimate the shock velocity, which can be derived from the [O III]/[O II] ratio as mentioned in section 3.2.3. Inspired by the work of Hester et al. (1983), the [O III]/ [O II] ratio can be used as an abscissa while the $[\mathrm{O} \mathrm{II}] / \mathrm{H} \alpha,[\mathrm{O} \mathrm{III}] / \mathrm{H} \alpha,[\mathrm{N} \mathrm{II}] / \mathrm{H} \alpha$ and $[\mathrm{S} \mathrm{II}] / \mathrm{H} \alpha$ can be used as the ordinate. This configuration allows putting a physical meaning on each axis, the abscissa, the shock velocity and the ordinates a mean to adjust the abundances. This convenient setup works only under some circumstances which are going to be explained in detail in this section.

Fig. 9 shows diagrams using the axes configuration described above, showing both the observations made with SITELLE (grey dots) taken from the ratio maps and complete shock models calculated with different abundances (coloured lines). We used a fixed pre-shock density of $20 \mathrm{~cm}^{-3}$ and a magnetic field of $1 \mu \mathrm{G}$ for all models. It has to be noted that a different pre-shock density would have little effect on the models shown in Fig. 9 as long as the selected value remains inside the given pre-shock density range measured in IC 443 from the [S II] ratio in section 3.2.4. The shock velocity chosen run from $70 \mathrm{~km} \mathrm{~s}^{-1}$ to $200 \mathrm{~km} \mathrm{~s}^{-1}$ varying from left to right on the diagram. By selecting the $[\mathrm{O} \mathrm{III}] /[\mathrm{O} \mathrm{II}]$ ratio, only fast shocks were selected at the expense of slower shocks. Therefore, the data points do not include all the observed spectra with SITELLE.

An interesting feature coming from these diagrams is how well the slope on the $[\mathrm{O} \mathrm{III}] / \mathrm{H} \alpha$ diagram is reproduced, clearly showing the relation between the intensity of [O III] and the shock velocity. On the contrary, the slopes on the other diagrams ( [O II $] / \mathrm{H} \alpha,[\mathrm{N}$ II $] / \mathrm{H} \alpha$ and $[\mathrm{S} \mathrm{II}] / \mathrm{H} \alpha$ ) are very low, meaning that these ratios are affected very little inside a narrow shock velocity range $\left(\approx 80\right.$ to $\left.100 \mathrm{~km} \mathrm{~s}^{-1}\right)$. This feature can be exploited in order to grossly estimate plausible abundance ranges of $\mathrm{O}, \mathrm{N}$ and $\mathrm{S}$, since the $[\mathrm{O} \mathrm{II}] / \mathrm{H} \alpha,[\mathrm{N} \mathrm{II}] / \mathrm{H} \alpha$ and $[\mathrm{S} \mathrm{II}] / \mathrm{H} \alpha$ vary linearly with abundances change. This method is by no means to be used to determine precisely the abundances in IC 443 . The variation of $[\mathrm{O} I \mathrm{II}] / \mathrm{H} \alpha,[\mathrm{N}$ II $] / \mathrm{H} \alpha$ and $[\mathrm{S} \mathrm{II}] / \mathrm{H} \alpha$ are most likely the result of an intricate combination of several other physical parameters and will be discussed in section 3.3. What can be said with reasonable certainty with these diagrams is that some abundances could be discarded. For example, an abundance of nitrogen with $\log (\mathrm{N} / \mathrm{H})=-$ 4.17 seems to be a little high; as for sulphur, $\log (\mathrm{S} / \mathrm{H})=-4.88$ seems a little too low for the shock velocity range considered. As for oxygen, it seems that a wide range of abundances could explain the observed emissions.

The derived abundances range derived from Fig. 9 are somewhat close the solar values determined by Asplund et al. (2009) with the exception of nitrogen and sulphur being too low with values evaluated at $\log (\mathrm{N} / \mathrm{H})=-4.17$ and $\log (\mathrm{S} / \mathrm{H})=-4.88$. As for the solar abundance of oxygen, its derived value of $\log (\mathrm{O} / \mathrm{H})=-3.31$ could explain the observed spectrum. The abundances adopted in this paper from helium to zinc (excepting $\mathrm{N}$ and $\mathrm{S}$ ) were taken from the compiled solar abundances of Asplund et al. (2009), Scott et al. (2015a), Scott et al. (2015b) and Grevesse et al. (2015). The abundance of nitrogen and sulphur were chosen from Fig. 9 fitting the model on the point clouds and the list of abundances used to compute all presented models is given in Table 2 . 
Table 3. Shock parameters used to compute a grid of 57032 models combined with abundances given in table 2 .

\begin{tabular}{lll}
\hline \hline Shock parameters & Value range & Interval \\
\hline Shock velocity & $(20-150) \mathrm{km} \mathrm{s}^{-1}$ & $1 \mathrm{~km} \mathrm{~s}^{-1}$ \\
Pre-shock density & $(14-60) \mathrm{cm}^{-3}$ & $2 \mathrm{~cm}^{-3}$ \\
Cut-off temperature & $(1000-20000) \mathrm{K}$ & $500 \mathrm{~K}$ \\
Magnetic field & $1 \mu \mathrm{G}$ for all model & \\
\hline
\end{tabular}

\subsection{Bi-shock models with multiple parameters}

Previous sections have demonstrated that many shock parameters are responsible for the observed spectra in IC 443. So far in this paper, as well as in previous studies, the observations were mainly compared with single shock models that include only one set of parameters. While this rather simplistic technique allows to derive important parameters, such as the shock velocity, pre-shock density and even approximate the abundances, it does not always explain all the observed spectra. Fig. 9 exposes several flaws when it comes to compare shock models with observations too simplistically. The most obvious one is the inability to reproduce all observations above $\log ([\mathrm{O} I I I] /[\mathrm{O} I \mathrm{II}]) \approx-0.22$. The presence of elevated ratios can be explained by the presence of incomplete shocks, as mentioned in section 3.2.3 (Fig. 5), which demonstrates the sensitivity of the $[\mathrm{OIII}] /[\mathrm{O} \mathrm{II}]$ ratio to shock completeness. However, it does not suffice to run a grid of truncated models in order to improve the match between the models and observations. Fig. 9 shows that several shock models with different abundances allow the overlapping of models over observations. While these diagrams allow approximating the plausible abundances in IC 443, it does not explain the reason for the point dispersion and therefore the origin of the great variety of line ratios observed in IC 443. The variation of abundances alone would not make sense. Ideally, each spectrum should be represented by one model with its very own set of parameters.

The filamentary structure in IC 443 is pretty intricate with many filaments criss-crossing, as viewed from the observer's point of view. Upon close inspection, many distinct interlaced filaments show different intensities for a given emission line. As we show in section 4, the receding and approaching components of the expanding bubble show very different morphological structures, suggesting that shock conditions on a given line of sight could be different and that the summation of both components on the same line of sight can lead to a spectrum that cannot be interpreted with the help of a single shock model. Therefore, many locations obviously required more than one shock model.

In an attempt to better reconcile models and observations, we have computed a grid of 57,032 shock models with the parameters and intervals listed in Table 3 . The shock velocity range was chosen in order to reflect the measurements presented in section 3.2.3 and the pre-shock densities with those derived in section 3.2.4. We assume that they are shocks with different ages, as mentioned in section 3.2.2. We also assume that the abundances do not vary significantly in the observed region and selected one set of abundances as determined in section 3.2.5.

To simulate the effect of multiple filaments on the same line of sight, we have to sum a given number of distinct shock models according to the number of components. Since we do not always have a clear idea about the exact shock parameters for each component, we can generate a new grid of models based on another grid using permutations. The total number of permutations of such shock models is given by the following equation:

$C(n, r)=\left(\begin{array}{l}n \\ r\end{array}\right)=\frac{(r+n-1) !}{r !(n-1) !}$

where $n$ is the total number of shock models available in a grid and $r$ the number of models to be summed, or in other words the number of components on the same line of sight. Fabry-Perot observations conducted by Ambrocio-Cruz et al. (2017) have indeed shown that up to four velocity components can be found along the line of sight at some positions in the nebula.

From equation 1, we see that the total number of permutations can become very large when $r$ is significantly larger than 2, depending on the total number of shock models available in a grid. Since our observations do not allow us to determine the exact number of components at every point in IC 443 and due to computational limitations, we choose a value of $r=2$. This leads to a total number of of $1,626,353,028$ models permutations which were systematically compared with every spectrum shown in Fig. 10 (gray points) made from 35,479 spectra taken from our ratio maps. In total, $5.3 \times 10^{13}$ comparisons where made and models who predicted all 10 line ratios presented in Fig. 10 with a margin of error smaller than 5\% were kept. The result is given on the same figure (black points) which shows 16,215 models overlapping the observations. About $45 \%$ of the selected spectra where fitted by our models. Most of the spectra can be represented by more then one model with different parameters leading to degeneracies.

Fig. 11 shows the frequency range of parameters that best fit the observations. These diagrams were made including the degeneracies. From that figure, we see that the spectra could be explained by the presences of shocks velocities range between 20 and $140 \mathrm{~km}$ $\mathrm{s}^{-1}$ with a clear preference toward velocity around $75 \mathrm{~km} \mathrm{~s}^{-1}$. Interestingly, there is a dip around that velocity where there should be a peak. This can be explained by the fact that the $[\mathrm{O} \mathrm{III]/[O} \mathrm{II]} \mathrm{ratio}$ quite steeply above $75 \mathrm{~km} \mathrm{~s}^{-1}$ as shown in Fig. 5. Any small error on the $[\mathrm{O} \mathrm{III}] /\left[\mathrm{O}_{\mathrm{II}}\right]$ ratio can easily "shift" the velocity on the other side of the dip as shown in Fig. 11. The same figure also shows a variety of cut-off temperatures, and thus shocks with different ages, favoring complete shocks. Models tend to favour pre-shock densities around $20 \mathrm{~cm}^{-3}$ but lower densities could not be excluded.

The most important result from Fig. 10 is that the combination of two shock models allows us to reproduce some observed spectra with $\log ([\mathrm{O} \mathrm{III}] /[\mathrm{O} \mathrm{II}]) \geqslant 0.60$, which were could not be explained with a simple model. This suggests that the individual spectra are indeed the combination of at least two components along the line of sight. Despite this success, some observation could not be reproduced. For example, there is a clear gap in the upper left for the [S II]/H $\alpha$ diagram and the lower left for the [N II]/[S II] diagram in Fig. 10 for which none of our summed models were able to fit the observations. However, single shock models can easily reproduce the observed ratios. It is probably the case with many other spectra for which one or more than three components can potentially provided a better fit to the spectra. However, more components will undeniably bring more degeneracies at which point the shock parameters found might lose their meaning and cannot provide additional informations.

It seems that one set of abundances is sufficient in order to reproduce the line intensities dispersion. In order words, that is no need to vary the abundances for specific spectrum in order to better fit the observations. We tested this with different abundances of $\mathrm{O}, \mathrm{N}$ and $\mathrm{S}$ around the ranges that can be inferred from Fig. 3.2.5 

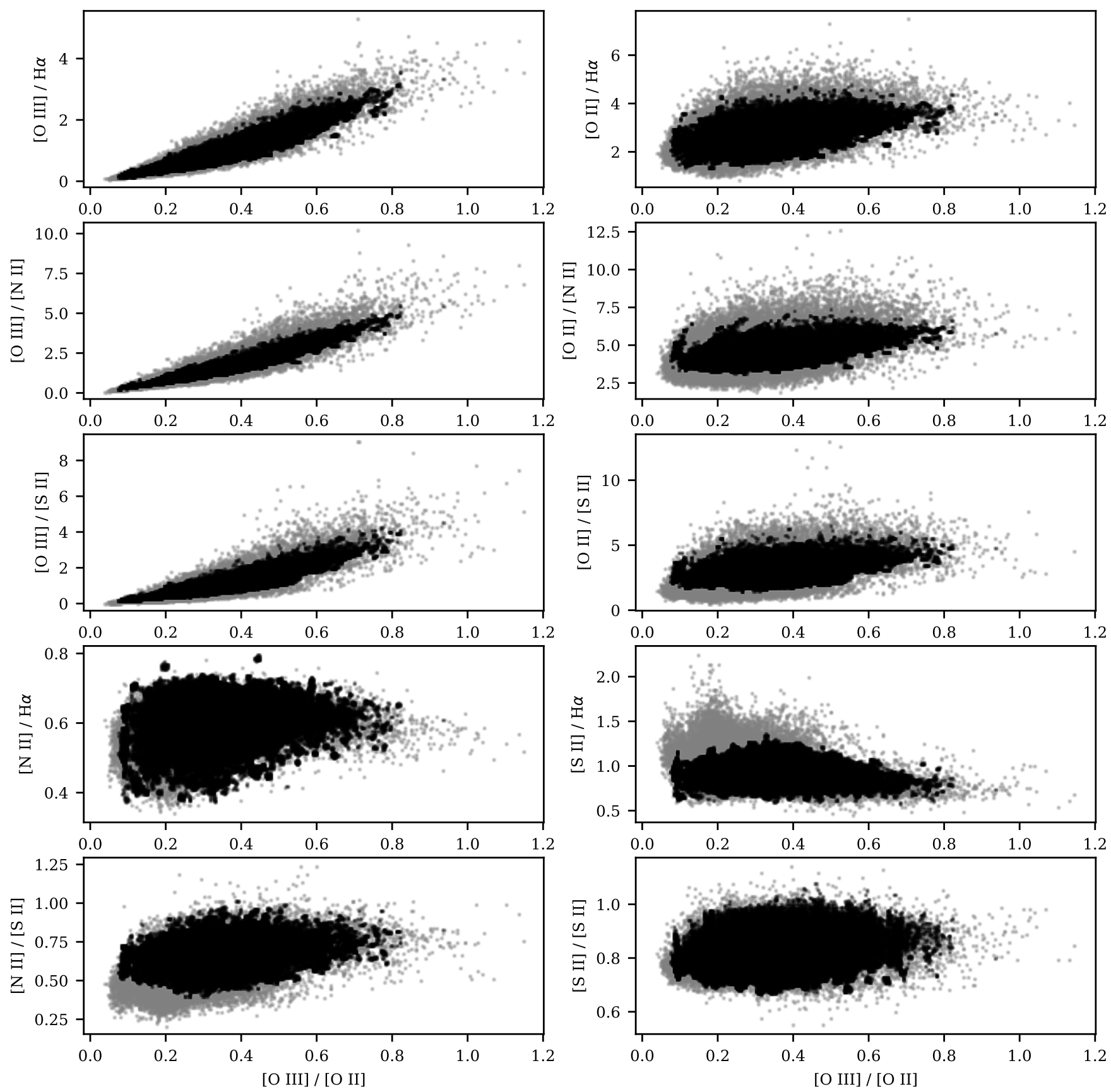

Figure 10. Bi-shock models in black on top of observations in gray. The combination of shock parameters for the shock velocity, cut-off temperature and pre-shock density are shown in a histogram in Fig.11. The abundances used are shown in Table 2

and saw no significant improvements. However, we noticed that an abundance of oxygen closer to solar value was preferable in order to better fit the observations.

We notice however high $[\mathrm{N}$ II] $/ \mathrm{H} \alpha$ ratios (Fig. A10 south of the field of view) which might suggest a region enriched with nitrogen. Since the $\left[\mathrm{O}_{\mathrm{III}}\right] /[\mathrm{O} \mathrm{II}]$ ratio cannot be obtained for these locations, we were unable to estimate a shock velocity and therefore accurately determine the nitrogen abundance. However, the absence of $[\mathrm{O} \mathrm{III}]$ for most of the location where $[\mathrm{N} \mathrm{II}] / \mathrm{H} \alpha$ is elevated suggests shocks slower than $75 \mathrm{~km} \mathrm{~s}^{-1}$. In that case, models calculated with a nitrogen abundance close to solar are unable to reproduce the observed $[\mathrm{N}$ II $] / \mathrm{H} \alpha$ and higher abundance must be considered. Figure 12 shows the variation of the $[\mathrm{N} \mathrm{II}] / \mathrm{H} \alpha$ ratios in function of different nitrogen abundances. It can been seen that for given $[\mathrm{N} I I] / \mathrm{H} \alpha$ ratio an accurate determination of the abundances is very sensitive to the shock velocity. When the velocity is unknown, multiple plausible abundances could be responsible for a unique ratio.

From figure A10 we measured a ratio of $[\mathrm{N} \mathrm{II}] / \mathrm{H} \alpha$ as high as 1.2. From figure 12 we can see that a shock velocity around 75 $\mathrm{km} \mathrm{s}^{-1}$ with an abundance of nitrogen of 3 times the solar could 

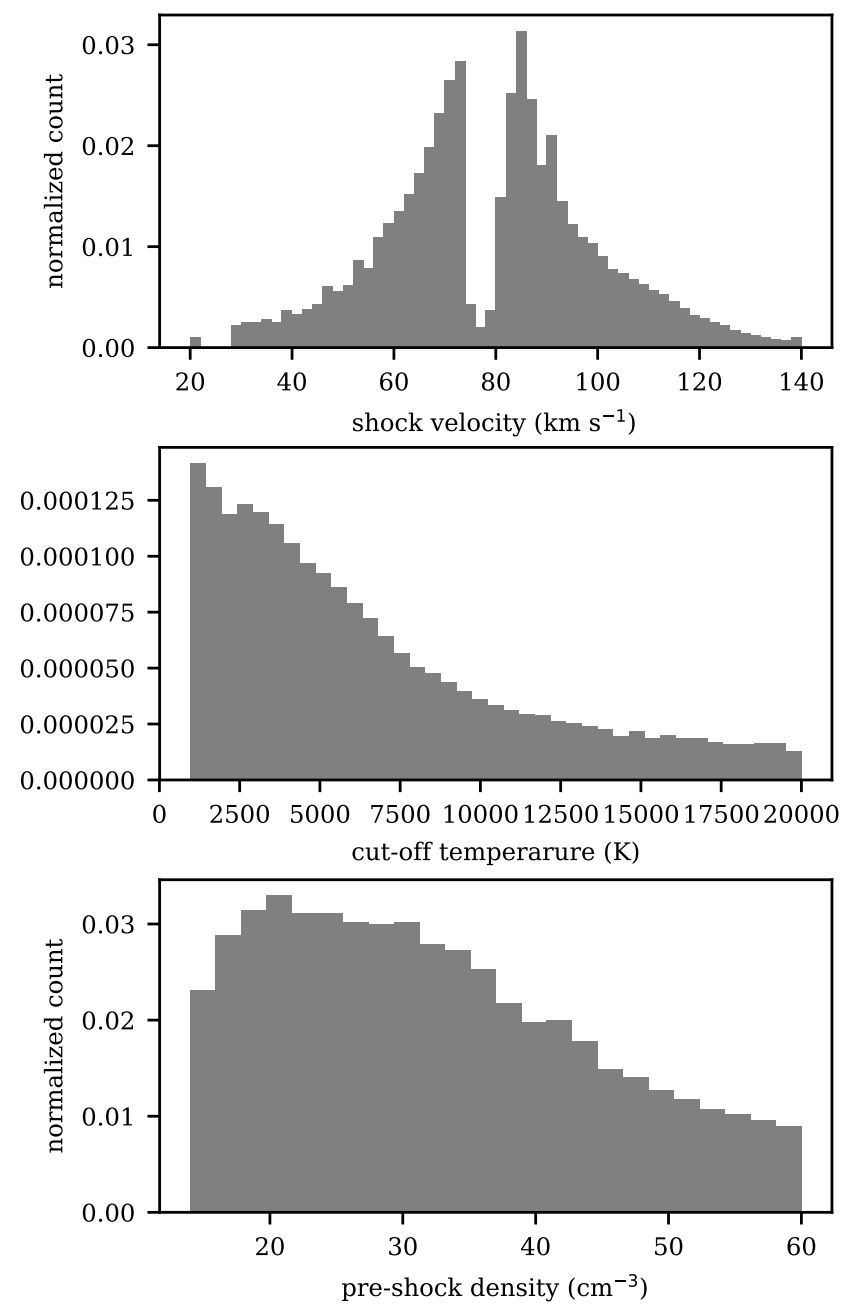

Figure 11. Histogram showing the distribution of the combination of two models for the shock velocity (top), cut-off temperature (middle) and preshock density (bottom) shown in Figure 10.

explain the observation. However, if the shock velocity is slower, a higher abundances of nitrogen must be considered.

The location of this enrichment in nitrogen is interesting since it is located toward the center of the nebula and might be ejecta from the progenitor which was blown outward by its stellar winds prior to its explosion.

\section{KINEMATICS}

A few measurements of the radial velocities across IC 443 have been obtained using long-slit spectroscopy (Lozinskaya \& Esipov 1969) and Fabry-Perot interferometer (Lozinskaya 1969; Pismis \& Rosado 1974; Lozinskaia 1979; Ambrocio-Cruz et al. 2017). The radial velocities measured by the various observations reported velocities ranging from approximately -180 to $+135 \mathrm{~km} \mathrm{~s}^{-1}$ and vary quite significantly across the nebula.

While our primary intent was not to study the kinematics (hence the relatively low spectral resolution of our cubes: 130 $\mathrm{km} / \mathrm{s} / \mathrm{channel}$ at $\mathrm{H} \alpha$ ), the high signal-to-noise of the SN3 cube, as

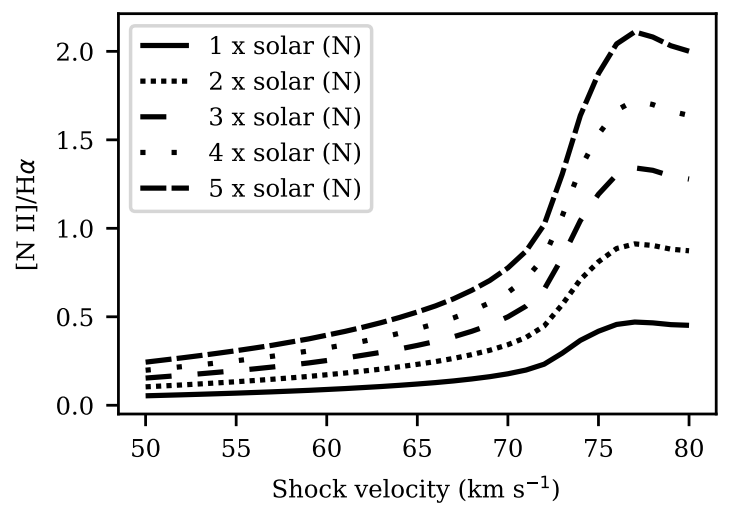

Figure 12. Variation of the $[\mathrm{N} \mathrm{II}] / \mathrm{H} \alpha$ ratio for different nitrogen abundances vs the shock velocity. $1 \times$ solar corresponds to a nitrogen abundance of $\log (\mathrm{N} / \mathrm{H})=-4.17$. The other abundances used to compute the curves are given in table 2

well as the relatively similar intensity of four emission lines within the limits of this filter have allowed us to separate the ionized gas into two distinct and morphologically very different components. Two approaches, leading to very similar results, were followed.

The first one is very simple: the spectral sampling and the expansion velocity of the nebula are such that emission from the nebula is visible on three consecutive frames of the data cube for each line: $\mathrm{H} \alpha$-red, $\mathrm{H} \alpha$-green and $\mathrm{H} \alpha$-blue; [N II] $\lambda 6584$-red, and so on. The line centroid (and hence maximum emission) does not always coincide with the middle frame and the balance between the red and the blue components is not identical from one line to the next. However, combining all the red frames together and doing the same for all the blue frames results in two emission-line maps sampling velocities separated by $260 \mathrm{~km} \mathrm{~s}^{-1}$. These images, which essentially sample the blue and red wings of all emission lines, are shown in Fig. 13, and a color composite is presented in Fig. 14 . While the upper left part of the nebula in this field looks pretty similar in the red and blue channels, the red and blue components of the lower right part, closer to the center of the supernova remnant and thus where the Doppler shift is expected to be larger, are dramatically different. While this method is relatively straightforward and provides a good glimpse at the three-dimensional structure of the nebula, it does not offer a quantitative distribution of the velocities at play.

We have therefore used ORCS to quantify this. Among the fitting procedures offered by ORCS, two are particularly adapted to the IC 443 data: as a first approach, the lines can be fitted using a sincgauss function (Martin et al. 2016), which is a convolution of the instrument line shape - a sinc - with a gaussian function characterizing the Doppler broadening of the line; or they can be fitted with two sinc components attributable to two distinct (approaching and receding) components. As expected, the sincgauss fit revealed a clear difference between the upper section of the field, where the sincgauss fit was not significantly different from a single sinc fit (given the low spectral resolution of our cube), and the lower right part, where a significant broadening - between 75 and $200 \mathrm{~km} \mathrm{~s}^{-1}$ was clearly detected. We have thus targeted a $7.3 \times 4.5$ arcmin region in the lower right corner of the field (see Fig. 13) to perform a two-component sinc fit. While ORCS allows to attribute different velocities to the individual lines, we chose to force all the lines present in the SN3 filter to share the same velocity. An example 
of these two fits on the spectrum of a single pixel is presented in Fig. 15, and the maps resulting from individual fits to the entire region are shown in Fig. 16. The result is strikingly similar to that using the simple approach described above. It is important to note that the limitations of this fitting technique become apparent in the upper left section of the blue component, where very subtle, but nevertheless present, stripes are visible. In this region, the width of the sincgauss function approaches $50 \mathrm{~km} \mathrm{~s}^{-1}$, one fourth of the instrumental line function's width. An histogram of the velocities resulting from the fits is presented in Fig. 17: the two components are clearly separated, by an average of $150 \mathrm{~km} \mathrm{~s}^{-1}$. While the receding component of the nebula presents a simple distribution, the approaching one is clearly bi-modal. There is also a very obvious difference in the morphologies of the two components (Fig. 16): the approaching side (very likely the front of the nebula, closer to us) presents small-scale honeycomb-like structures, while the other side (presumably the back, more distant) is smoother and more regular. This dichotomy very likely reflects the structure of the interstellar medium in which the blast wave is expanding (such as the pre-shock density). We also note that the combined flux of all the SN3 emission lines is twice as large in the red component as in the blue one.

While ORCS also provides the flux in the individual lines for both components, we do not wish to over-interpret our rather low spectral-resolution data at this time, but note that a cube with a higher spectral resolution could provide a means to determine the physical properties of both components individually. In the previous sections, we have demonstrated that a single shock model could not reproduce the observed line ratios. The different morphologies of the red and blue components indeed suggest a dichotomy in their shock properties.

\section{CONCLUDING REMARKS}

We used the optical imaging Fourier transform spectrometer SITELLE to obtain multi-spectral cubes of the north-eastern part of IC 443. From these cubes, we generated maps allowing the investigation of the spatial variations in spectral line intensity of seven emission lines: $\mathrm{H} \alpha, \mathrm{H} \beta$, [O II] $\lambda 3726+3729$, [O III] $\lambda 5007$, [N II] $\lambda 6583$ and [S II] $\lambda 6716,6731$. We compared the observations with shock models computed with MAPPINGS and estimates important physical properties found in IC 443. Specific results are as follows:

(i) The reddening has been measured from the $\mathrm{H} \alpha / \mathrm{H} \beta$ ratio for the observed region and found to vary significantly at its surface with $\mathrm{E}(\mathrm{B}-\mathrm{V})$ of $0.8-1.1$.

(ii) The electron density observed in the filaments ranges from less than 100 to $2500 \mathrm{~cm}^{-3}$ with higher densities found at the extremity of the north-eastern part of IC 443 .

(iii) The line intensities vary quite significantly across the field of view and can be traced to the presence of different shock velocities at different stage of recombination. High $\left[\mathrm{O}_{\mathrm{III}}\right] / \mathrm{H} \beta$ and $[\mathrm{O}$ II $] / \mathrm{H} \beta$ ratios are higher towards the direction where the shock front is propagating, due to the presence of incomplete shocks. High $[\mathrm{S} \mathrm{II}] / \mathrm{H} \alpha$ with no emissions in [O III] toward the center of the observed region indicates the location of shocks with velocity slower then $40 \mathrm{~km} \mathrm{~s}^{-1}$.

(iv) Shock models with a unique set of parameters are unable to reproduce the great majority of the observed emissions. The summation of two shock models (bi-models) with different shock parameters allow a better prediction of the variability of the observed emission line ratios. Bi-models comparisons allow to constraint the probable shock condition inside IC 443 for the observed region. The most probable shock velocities varying from 20 to $150 \mathrm{~km} \mathrm{~s}^{-1}$ with $75 \mathrm{~km} \mathrm{~s}^{-1}$ being the most common velocity found, the preshock density between $20-60 \mathrm{~cm}^{-3}$ favouring lower densities and shocks at different stages of recombination as suggested by wide range of the $[\mathrm{O} I I I] / \mathrm{H} \beta$ ratios.

(v) A single set of abundances close to solar values combined with varying shock velocity, pre-shock density and shocks with different ages are sufficient to explained the measured ratios.

(vi) Despite the modest spectral resolution of the SN3 cube, we were able to separate the approaching and receding components of the expanding nebula. These two components harbor very different morphological structures, probably resulting from different properties of the interstellar medium in which the blast wave propagates. Higher spectral resolution data cubes across the visible range with SITELLE would allow us to better probe the physical condition on both sides of the nebula.

\section{ACKNOWLEDGEMENTS}

Based on observations obtained with SITELLE, a joint project of Université Laval, ABB, Université de Montréal, and the CanadaFrance-Hawaii Telescope (CFHT) which is operated by the National Research Council of Canada, the Institut National des Sciences de l'Univers of the Centre National de la Recherche Scientifique of France, and the University of Hawaii. The authors wish to recognize and acknowledge the very significant cultural role that the summit of Mauna Kea has always had within the indigenous Hawaiian community. We are most grateful to have the opportunity to conduct observations from this mountain. AA thanks CONACyT-CB2015-254132 project for supporting his postdoc position at IA-UNAM. Models were performed using computers from projects DGAPA/PAPIIT-107215 and CONACyT-CB2015254132. LD is grateful to the Natural Sciences and Engineering Research Council of Canada, the Fonds de Recherche du Québec, and the Canada Foundation for Innovation for funding. LD is very grateful to the Department of Physics and Astronomy, University of Hawaii Hilo, as well as the CFHT, for having provided him with an ideal and very enjoyable working environment during his sabbatical stay on the Big Island.

All computations from data reduction to analysis were conducted using open source software : PYTHON programming language and the GNU Data Language. Special thanks to the people who developed and maintain vital libraries used in this work: NUMPY \& SCIPY (van der Walt et al. 2011), CYTHON (Behnel et al. 2011) and MATPLOTLIB (Hunter 2007). This work also made extensive use of the GNU PARALLEL shell tool (Tange 2011).

\section{REFERENCES}

Abdo A. A., et al., 2010, ApJ, 712, 459

Ackermann M., et al., 2013, Science, 339, 807

Albert J., et al., 2007, ApJ, 664, L87

Allen M. G., Groves B. A., Dopita M. A., Sutherland R. S., Kewley L. J., 2008, ApJS, 178, 20

Ambrocio-Cruz P., Rosado M., de la Fuente E., Silva R., Blanco-Piñon A., 2017, MNRAS, 472, 51

Asplund M., Grevesse N., Sauval A. J., Scott P., 2009, ARA\&A, 47, 481

Behnel S., Bradshaw R., Citro C., Dalcin L., Seljebotn D. S., Smith K., 2011, Computing in Science Engineering, 13, 31 

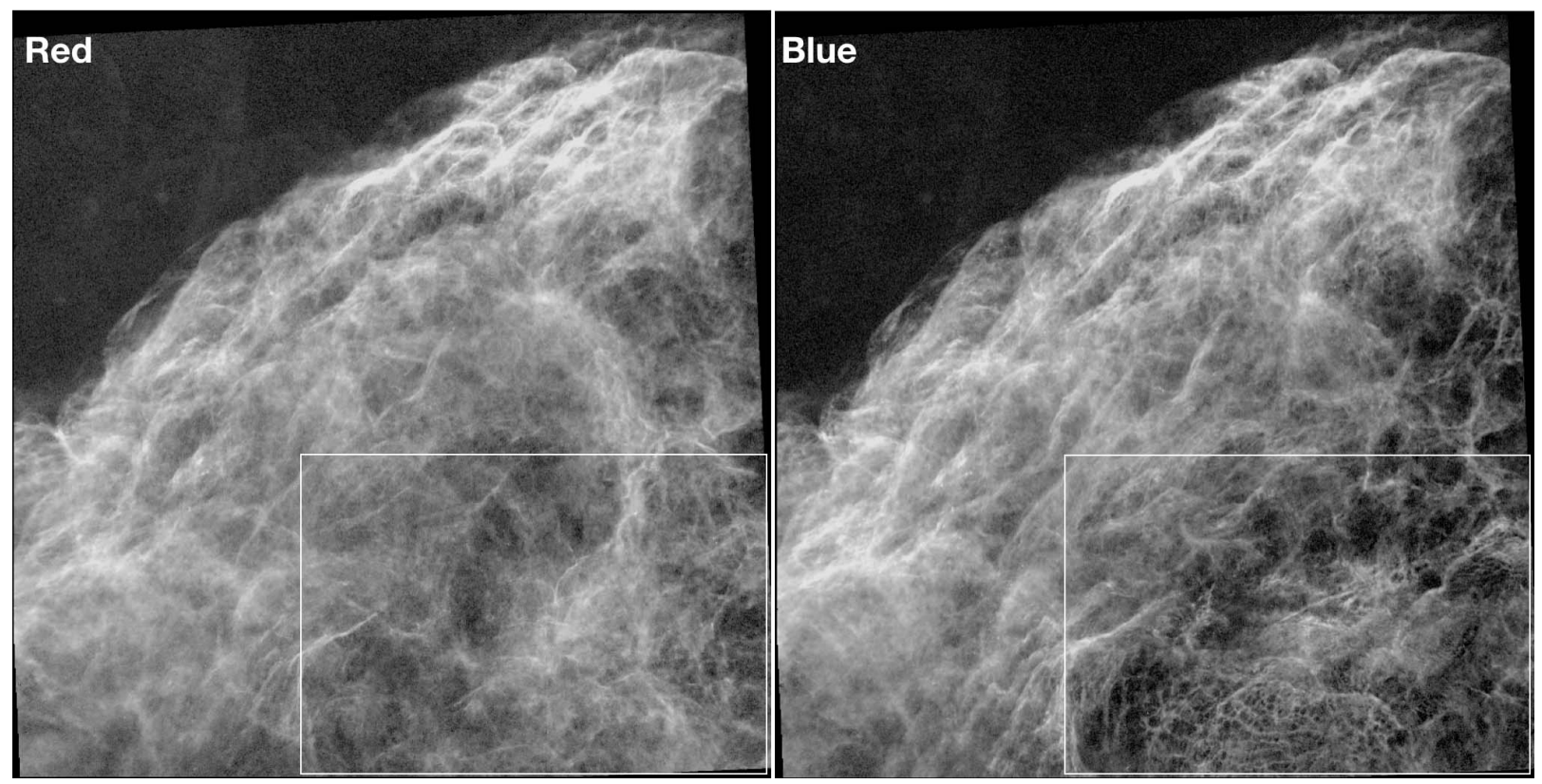

Figure 13. Red and blue components of all emission lines present in the SN3 filter, obtained by selecting the frames in the data cube corresponding to the wings of the lines (see text). The region which was analyzed kinematically with ORCS is indicated by a white rectangle.

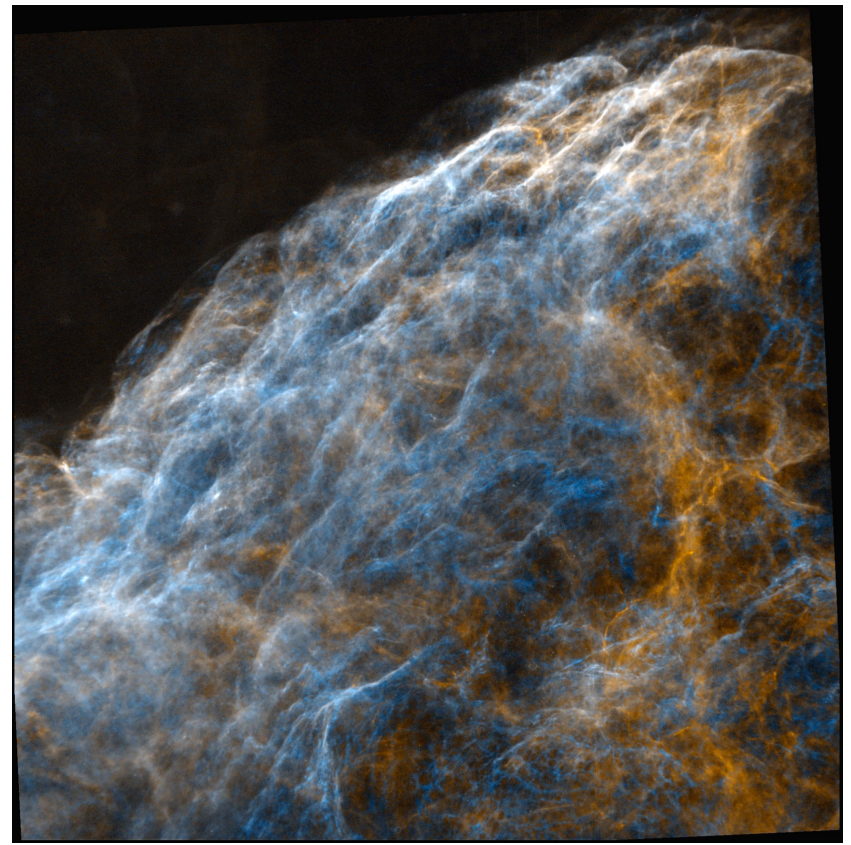

Figure 14. Color-coded combination of the two images shown in Fig. 13.

Binette L., Dopita M. A., Dodorico S., Benvenuti P., 1982, A\&A, 115, 315 Binette L., Dopita M. A., Tuohy I. R., 1985, ApJ, 297, 476

Bocchino F., Miceli M., Troja E., 2009, A\&A, 498, 139

Braun R., Strom R. G., 1986, A\&A, 164, 193

Burton M. G., Geballe T. R., Brand P. W. J. L., Webster A. S., 1988, MNRAS, 231, 617

Contini M., Shaviv G., 1982, Ap\&SS, 85, 203

Del Zanna G., Dere K. P., Young P. R., Landi E., Mason H. E., 2015, A\&A, 582, A56

Dere K. P., Landi E., Mason H. E., Monsignori Fossi B. C., Young P. R., 1997, A\&AS, 125, 149
Dickman R. L., Snell R. L., Ziurys L. M., Huang Y.-L., 1992, ApJ, 400, 203 Dopita M. A., 1976, ApJ, 209, 395

Dopita M. A., 1977, ApJS, 33, 437

Dopita M. A., 1978, ApJS, 37, 117

Dopita M. A., 1979, ApJS, 40, 455

Dopita M. A., Sutherland R. S., 1996, ApJS, 102, 161

Dopita M. A., Mathewson D. S., Ford V. L., 1977, ApJ, 214, 179

Dopita M. A., Dodorico S., Benvenuti P., 1980, ApJ, 236, 628

Dopita M. A., Binette L., Schwartz R. D., 1982, ApJ, 261, 183

Dopita M. A., Sutherland R. S., Nicholls D. C., Kewley L. J., Vogt F. P. A., 2013, ApJS, 208, 10

Drissen L., et al., 2019, MNRAS, 485, 3930

Esipov V. F., Kaplan S. A., Lozinskaya T. A., Podstrigach T. S., 1972, Soviet Ast., 16, 81

Fang J., Yu H., Zhu B.-T., Zhang L., 2013, MNRAS, 435, 570

Fesen R. A., Kirshner R. P., 1980, ApJ, 242, 1023

Fitzpatrick E. L., 1999, PASP, 111, 63

Grevesse N., Scott P., Asplund M., Sauval A. J., 2015, A\&A, 573, A27

Groves B. A., Dopita M. A., Sutherland R. S., 2004a, ApJS, 153, 9

Groves B. A., Dopita M. A., Sutherland R. S., 2004b, ApJS, 153, 75

Hester J. J., Parker R. A. R., Dufour R. J., 1983, ApJ, 273, 219

Hezareh T., Wiesemeyer H., Houde M., Gusdorf A., Siringo G., 2013, A\&A, 558, A45

Hunter J. D., 2007, Computing In Science \& Engineering, 9, 90

Lee J.-J., Koo B.-C., Yun M. S., Stanimirović S., Heiles C., Heyer M., 2008, AJ, 135, 796

Lozinskaia T. A., 1979, A\&A, 71, 29

Lozinskaya T. A., 1969, Soviet Ast., 13, 192

Lozinskaya T. A., Esipov V. F., 1969, Soviet Ast., 12, 913

Luridiana V., Morisset C., Shaw R. A., 2012, in IAU Symposium. pp 422 423, doi:10.1017/S1743921312011738

Luridiana V., Morisset C., Shaw R. A., 2015, A\&A, 573, A42

Martin T., Drissen L., 2017, preprint, (arXiv: 1706.03230)

Martin T., Drissen L., Joncas G., 2015, in Taylor A. R., Rosolowsky E., eds, Astronomical Society of the Pacific Conference Series Vol. 495, Astronomical Data Analysis Software an Systems XXIV (ADASS XXIV). p. 327

Martin T. B., Prunet S., Drissen L., 2016, MNRAS, 463, 4223

Olbert C. M., Clearfield C. R., Williams N. E., Keohane J. W., Frail D. A., 2001, ApJ, 554, L205 

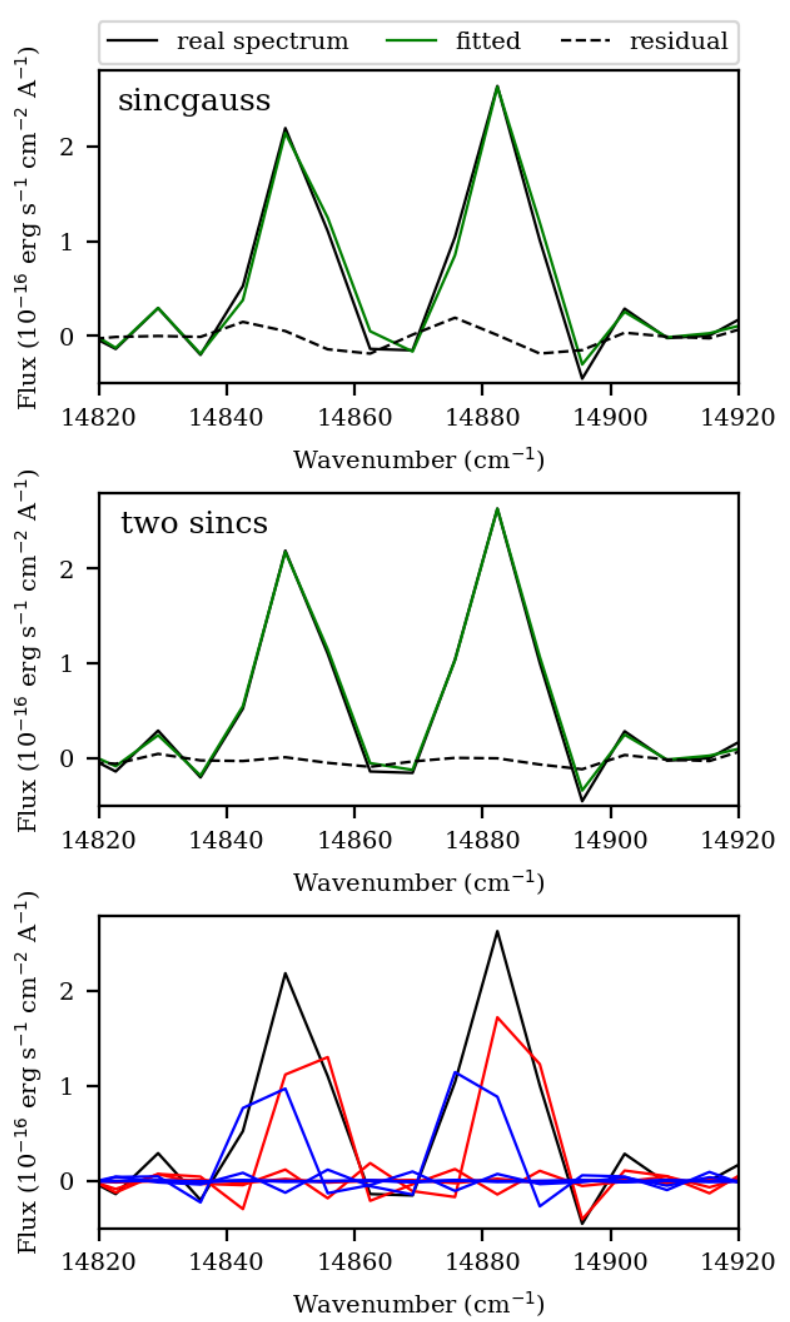

Figure 15. Example of the fitting functions used by ORCS, for the [S II] 6717,31 doublet in a single pixel. On the top left panel, a sincgauss function was tried, returning a velocity dispersion, $\sigma=120 \mathrm{~km} / \mathrm{s}$. On the top right, the result of two sinc functions. The lower panel shows the two components.

Osterbrock D. E., 1958, PASP, 70, 180

Parker R. A. R., 1964, ApJ, 139, 493

Petre R., Szymkowiak A. E., Seward F. D., Willingale R., 1988, ApJ, 335, 215

Pismis P., Rosado M., 1974, Rev. Mex. Astron. Astrofis., 1, 121

Raymond J. C., 1979, ApJS, 39, 1

Raymond J. C., Hartmann L., Black J. H., Dupree A. K., Wolff R. S., 1980, ApJ, 238, 881

Raymond J. C., Hester J. J., Cox D., Blair W. P., Fesen R. A., Gull T. R., 1988, ApJ, 324, 869

Rho J., Jarrett T. H., Cutri R. M., Reach W. T., 2001, ApJ, 547, 885

Scott P., et al., 2015a, A\&A, 573, A25

Scott P., Asplund M., Grevesse N., Bergemann M., Sauval A. J., 2015b, A\&A, 573, A26

Shull J. M., McKee C. F., 1979, ApJ, 227, 131

Snell R. L., Hollenbach D., Howe J. E., Neufeld D. A., Kaufman M. J., Melnick G. J., Bergin E. A., Wang Z., 2005, ApJ, 620, 758

Su Y., Fang M., Yang J., Zhou P., Chen Y., 2014, ApJ, 788, 122

Sutherland R. S., Dopita M. A., 1993, ApJS, 88, 253

Sutherland R. S., Dopita M. A., 2017, ApJS, 229, 34

Swartz D. A., et al., 2015, ApJ, 808, 84

Tange O., 2011, The USENIX Magazine, 36, 42

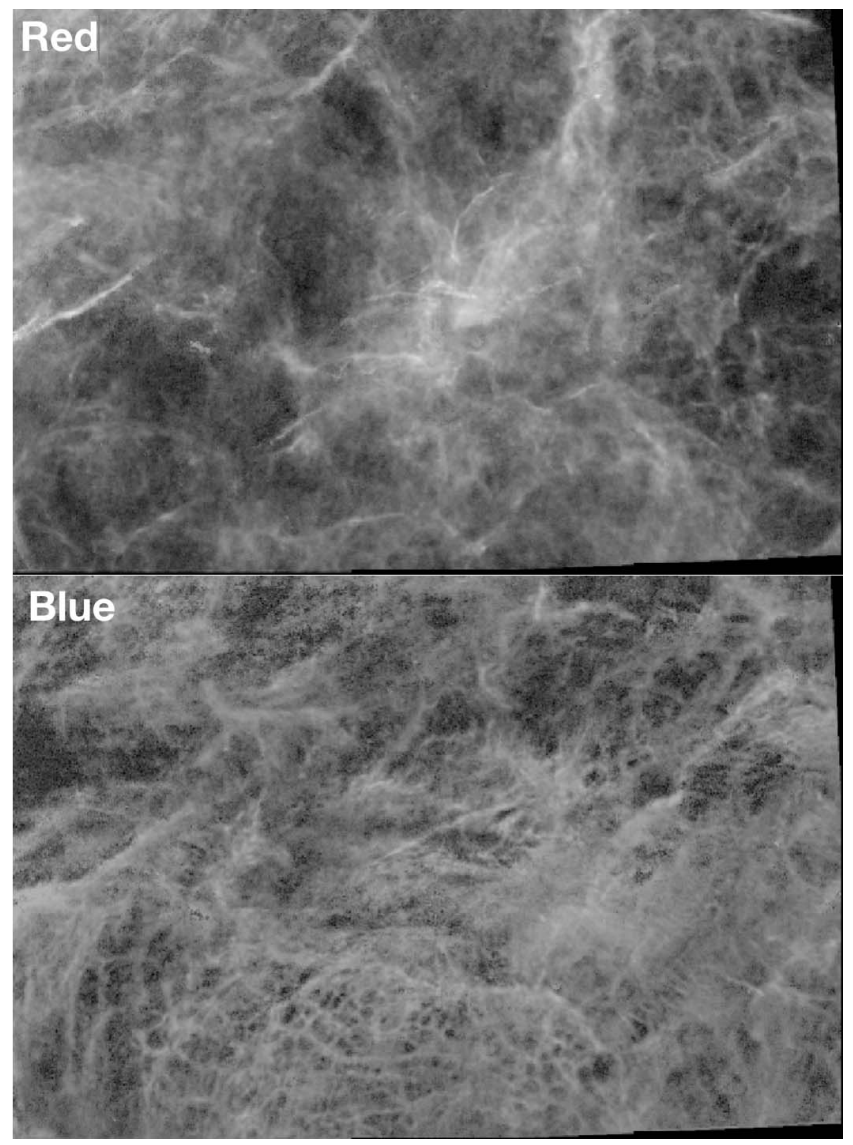

Figure 16. Red and blue components in the SW region $\left(7.3^{\prime} \times 4.5^{\prime}\right)$ of the SITELLE field of view, as extracted with ORCS (see text). The similarity between these images and Fig. 13 is striking. The limitations of this method are however visible in the upper left corner of the blue component image, where low-amplitude fringes can be seen.

Troja E., Bocchino F., Reale F., 2006, ApJ, 649, 258

Troja E., Bocchino F., Miceli M., Reale F., 2008, A\&A, 485, 777

Weedman D. W., 1968, PASP, 80, 314

Welsh B. Y., Sallmen S., 2003, A\&A, 408, 545

van Dishoeck E. F., Jansen D. J., Phillips T. G., 1993, A\&A, 279, 541

van der Walt S., Colbert S. C., Varoquaux G., 2011, Computing in Science

Engineering, 13, 22

\section{APPENDIX A: FIGURES}

This paper has been typeset from a $\mathrm{T}_{\mathrm{E}} \mathrm{X} / \mathrm{LT}_{\mathrm{E}} \mathrm{X}$ file prepared by the author. 


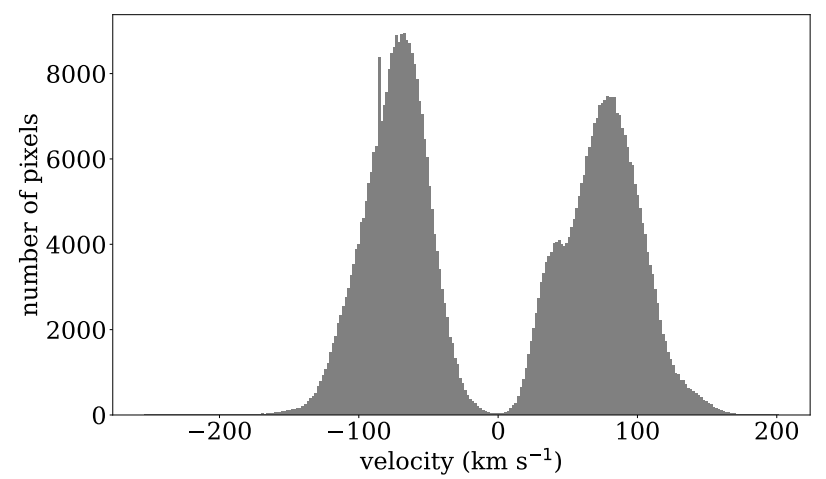

Figure 17. Histogram of the velocities obtained with ORCS in the region shown in Fig. 16. 


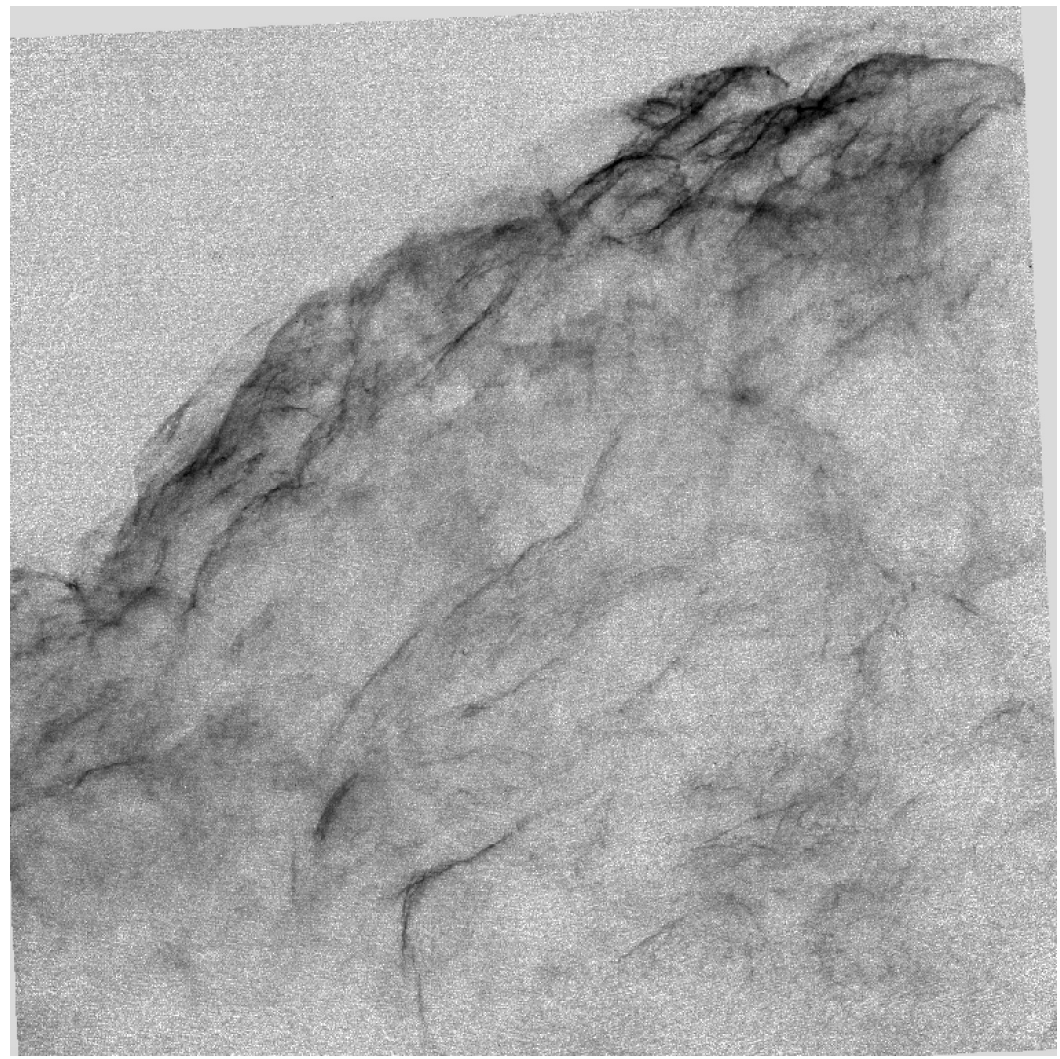

Figure A1. The observed region in $[\mathrm{O}$ II $] \lambda 3727+\lambda 3729$. FOV is $11^{\prime} \times 11^{\prime}$ with North at the top and East to the left.

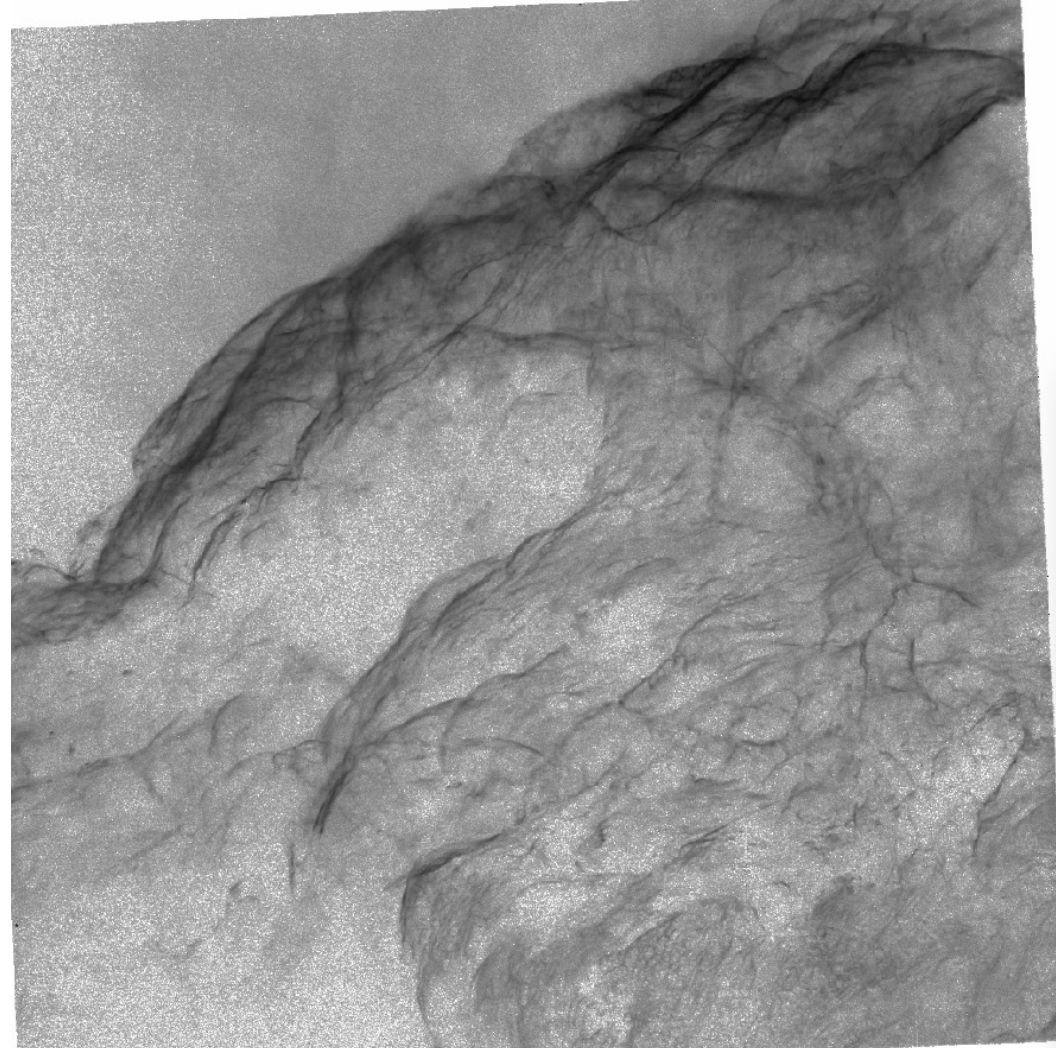

Figure A2. Same as Fig. A1, for [O III] $\lambda 5007$. 


\section{Alarie \& Drissen}

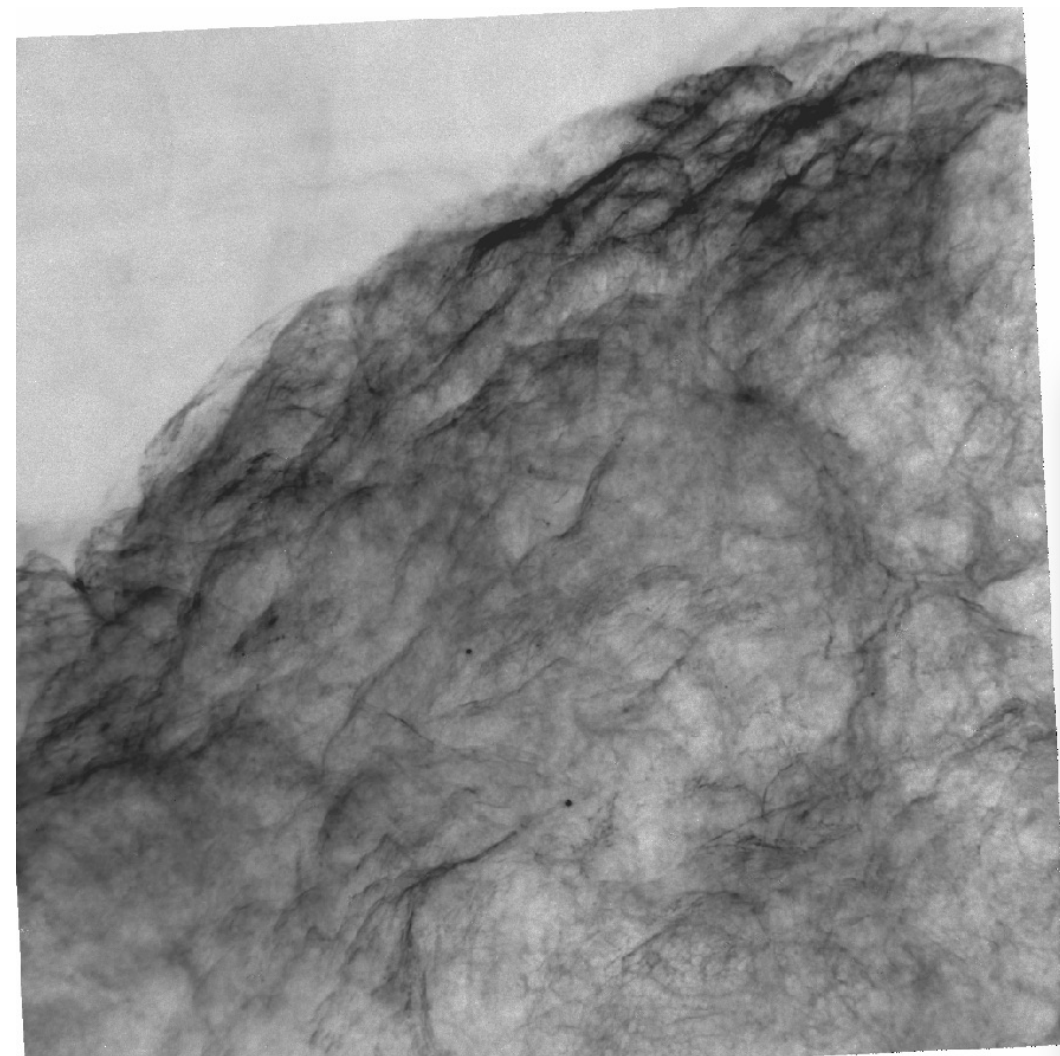

Figure A3. Same as Fig. A1, for $\mathrm{H} \alpha$. Note the presence of two emission-line stars near the central part of the image.

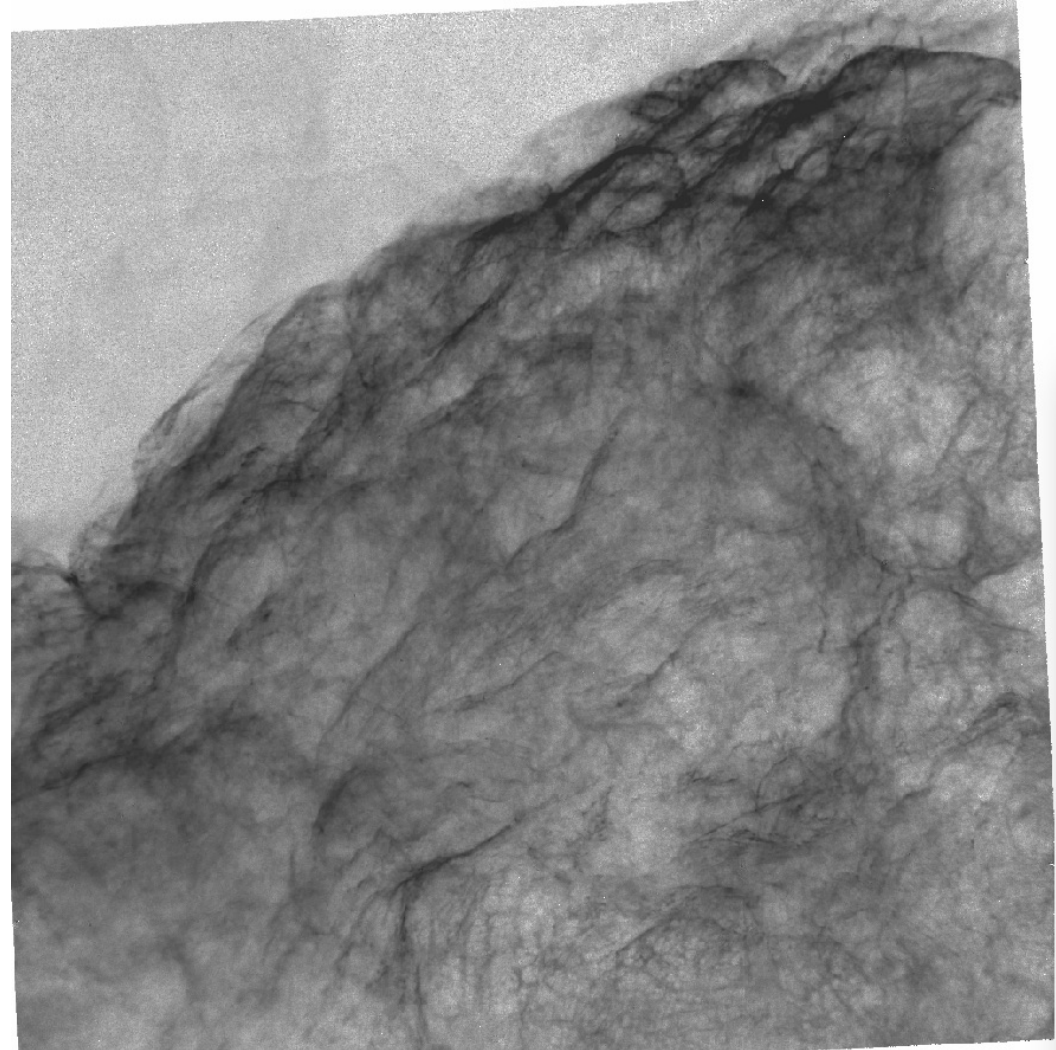

Figure A4. Same as Fig. A1, for [N II] $\lambda 6583$. 


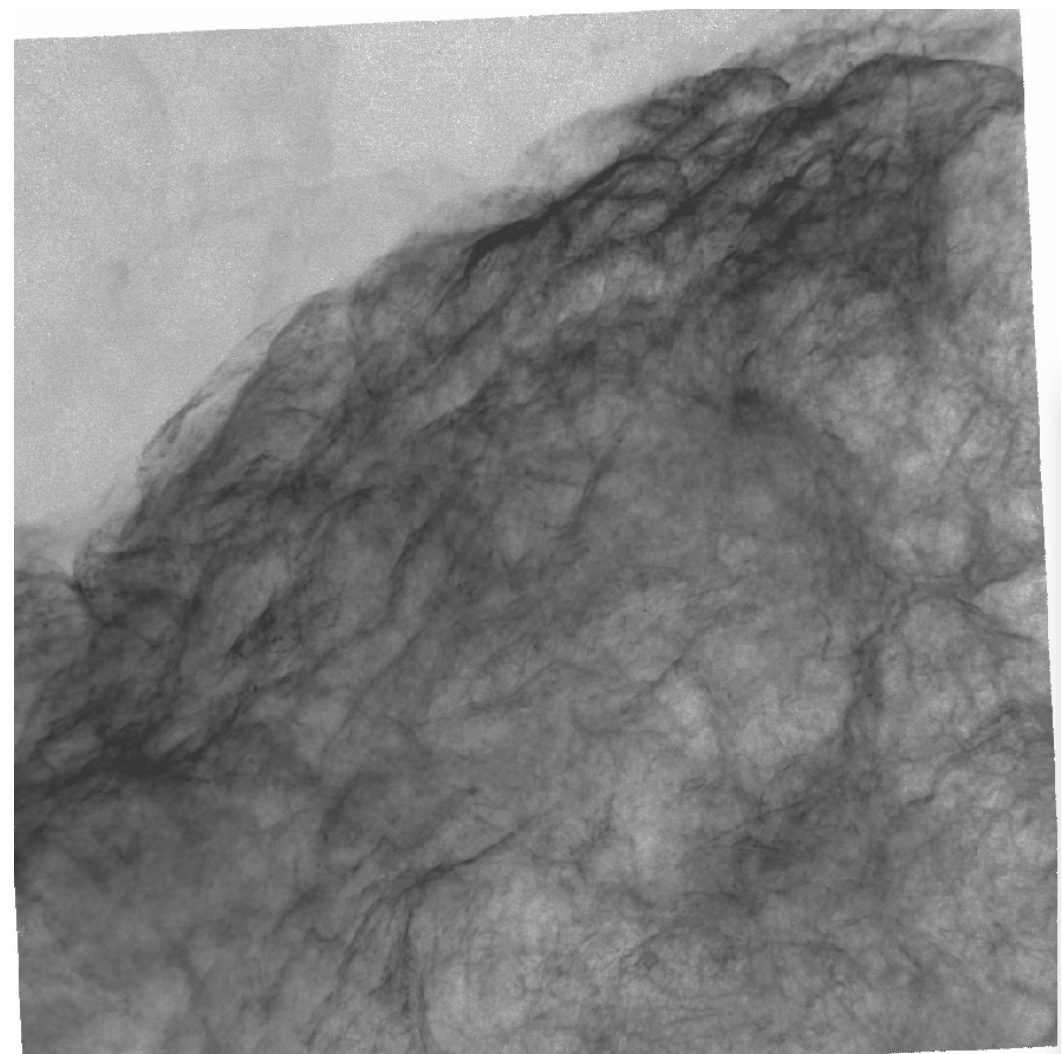

Figure A5. Same as Fig. A1, for $[\mathrm{S}$ II] $\lambda 6716+\lambda 6731$.

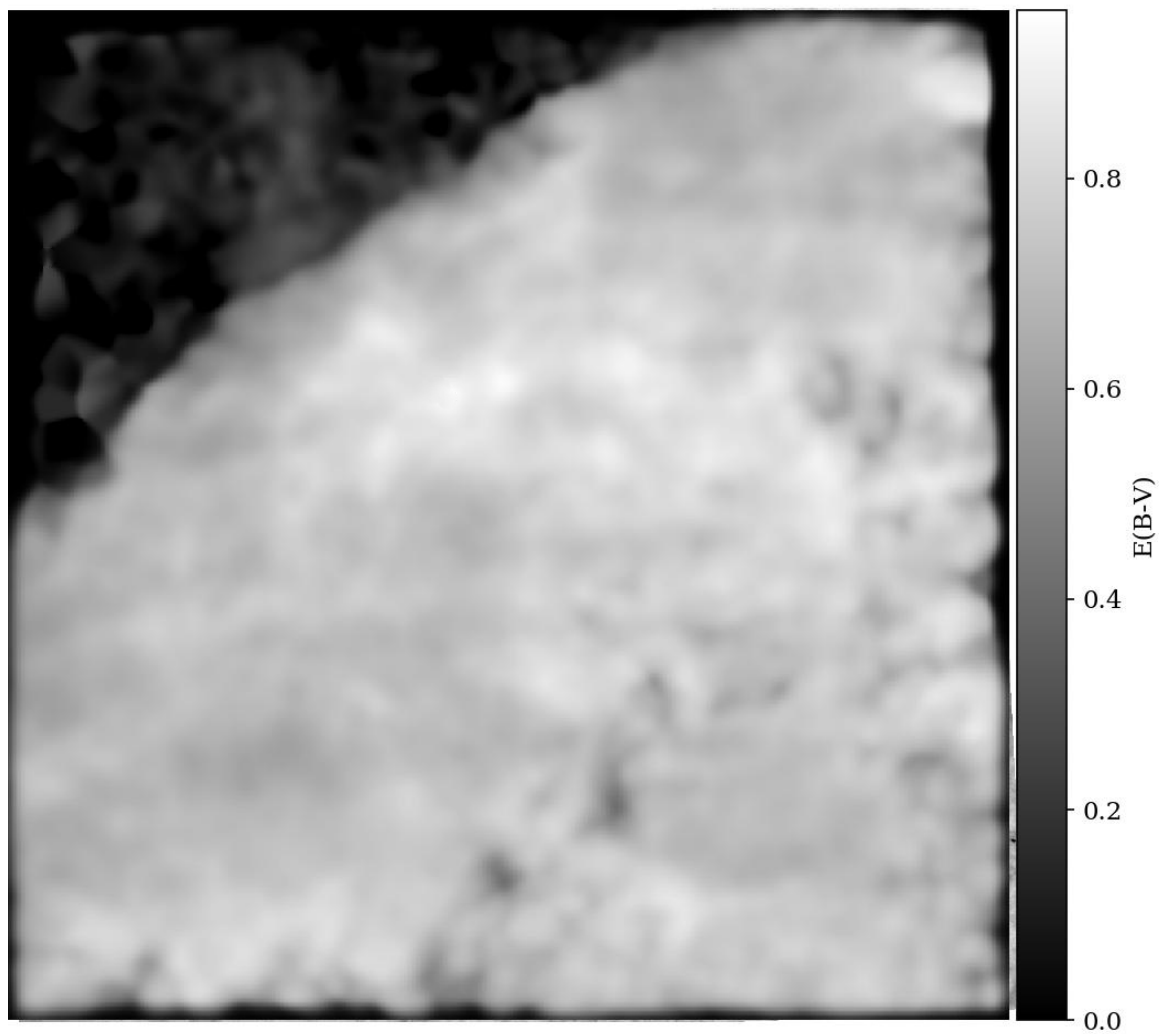

Figure A6. Extinction map of north-eastern part of IC 443 using the $\mathrm{H} \alpha / \mathrm{H} \beta$ line ratio. The map was smoothed with a Gaussian distribution with $\sigma=5.0$ arcsec. 


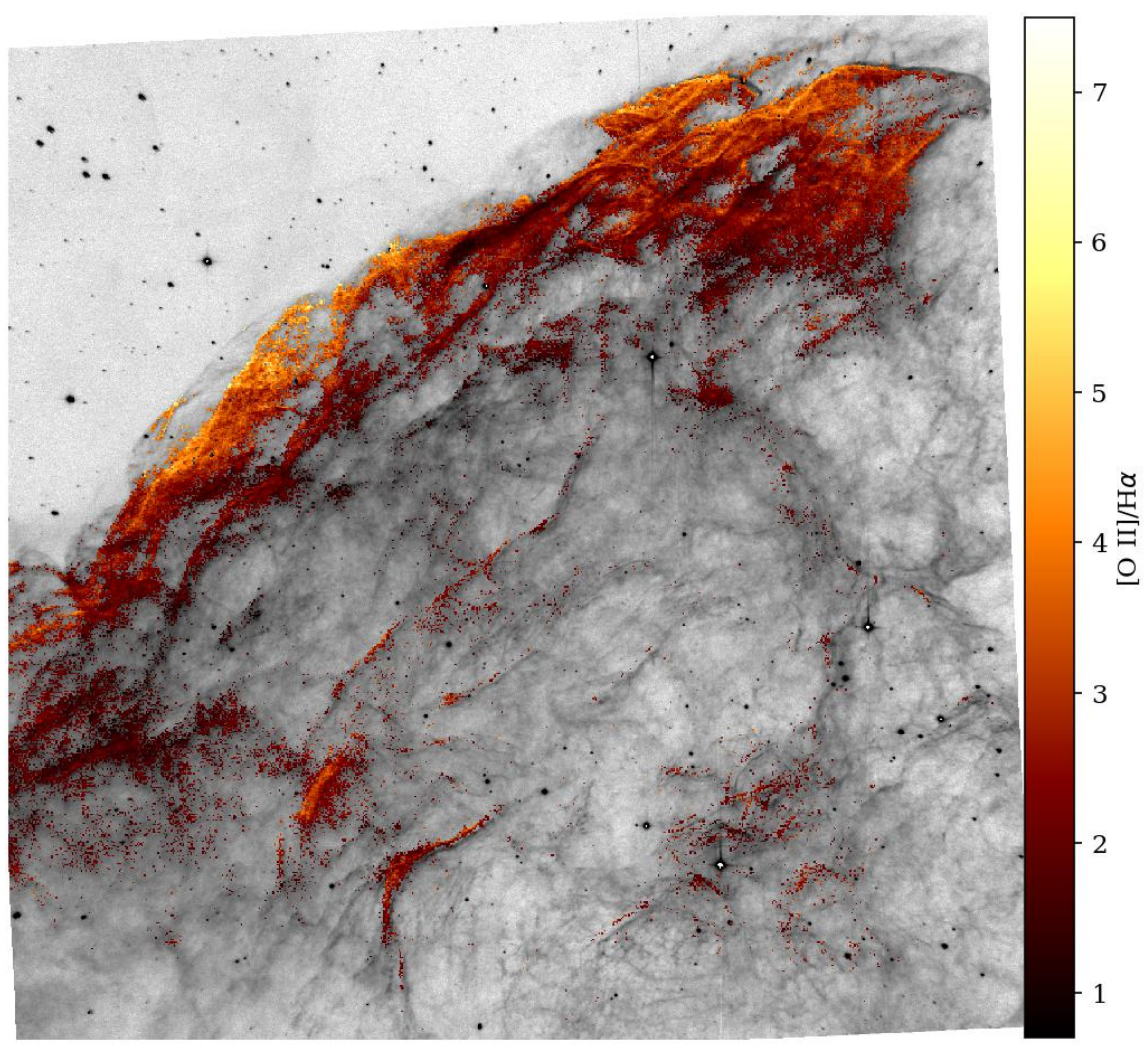

Figure A7. Map of the $[\mathrm{O}$ II $] \lambda 3727+\lambda 3729 / \mathrm{H} \alpha$ line ratio. Background image : $\mathrm{H} \alpha$ emission.

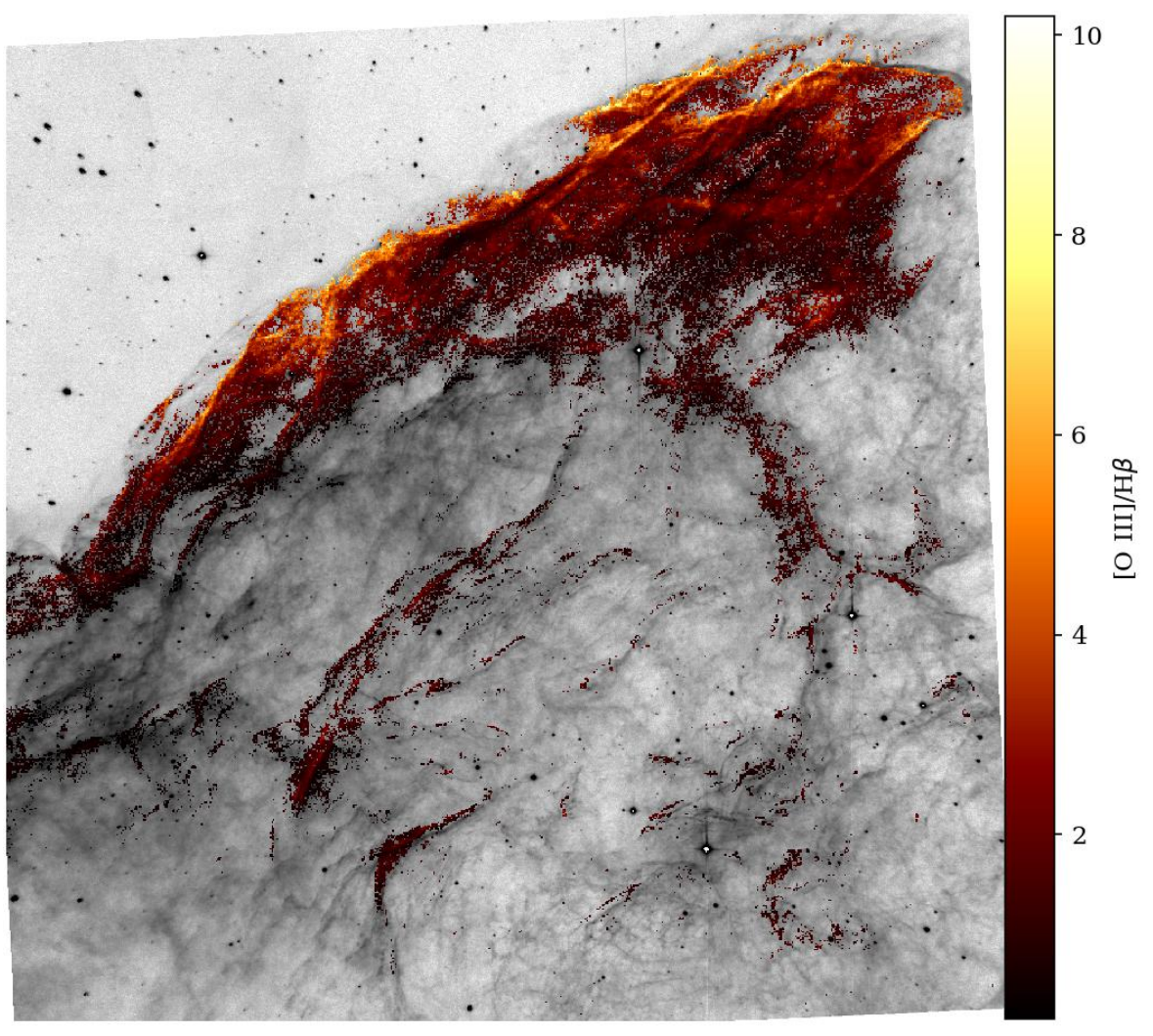

Figure A8. Same as Fig. A7, for $[\mathrm{O}$ III $] \lambda 5007 / \mathrm{H} \beta$. 


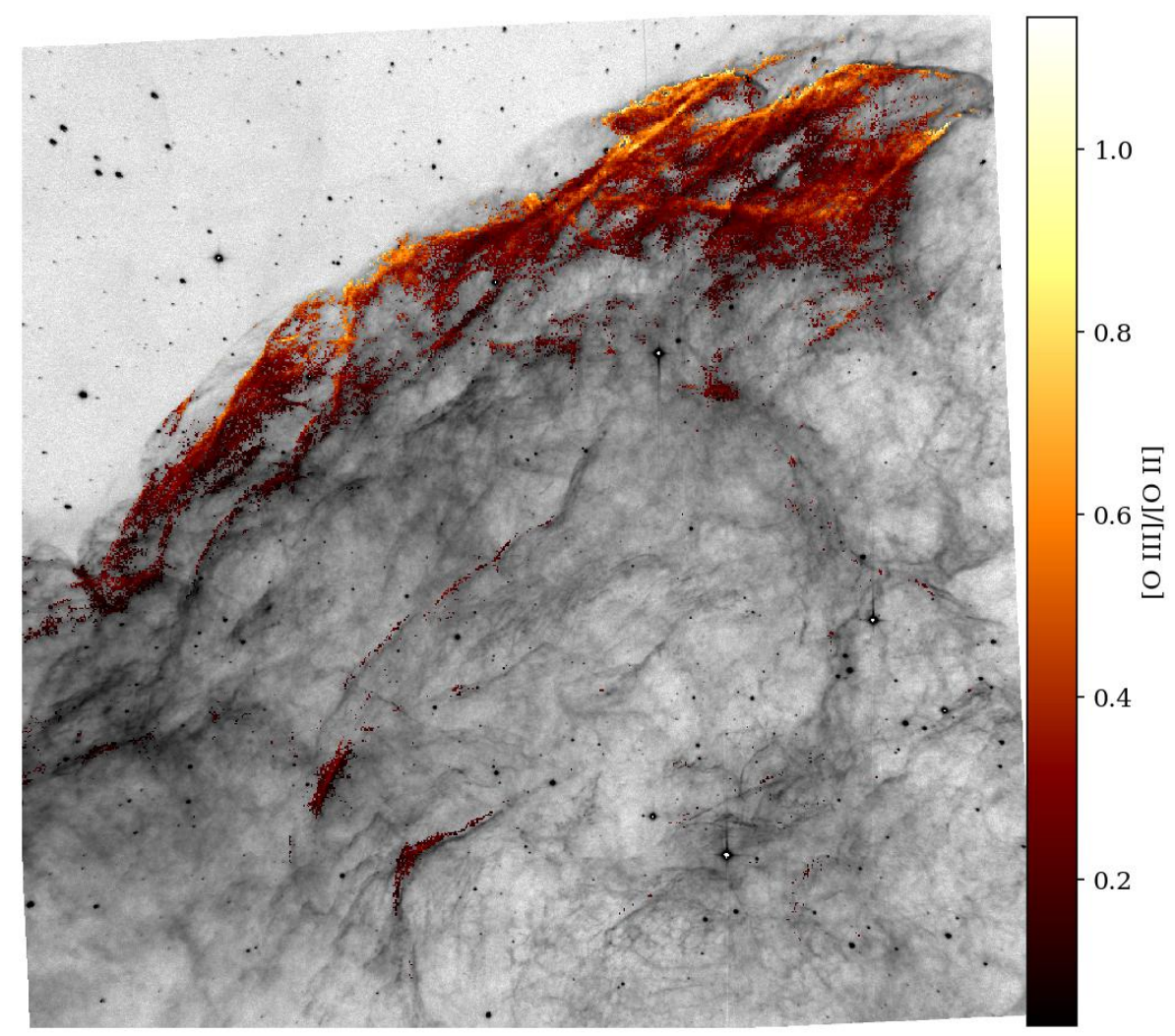

Figure A9. Same as Fig. A7, for [O III] $\lambda 5007 /[\mathrm{O}$ II $] \lambda 3727+\lambda 3729$.

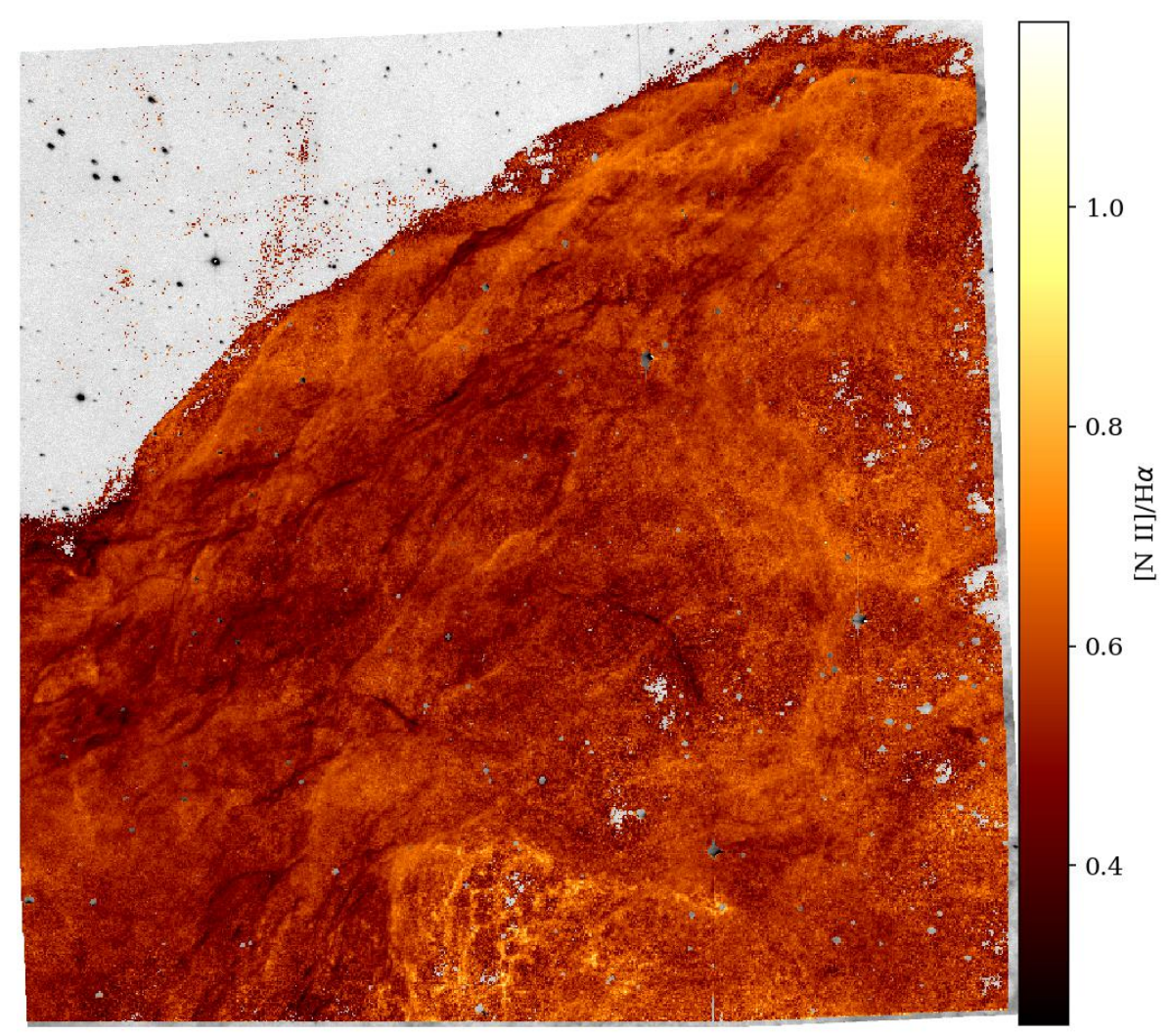

Figure A10. Same as Fig. A7, for $[\mathrm{N} I I] \lambda 6583 / \mathrm{H} \alpha$. 


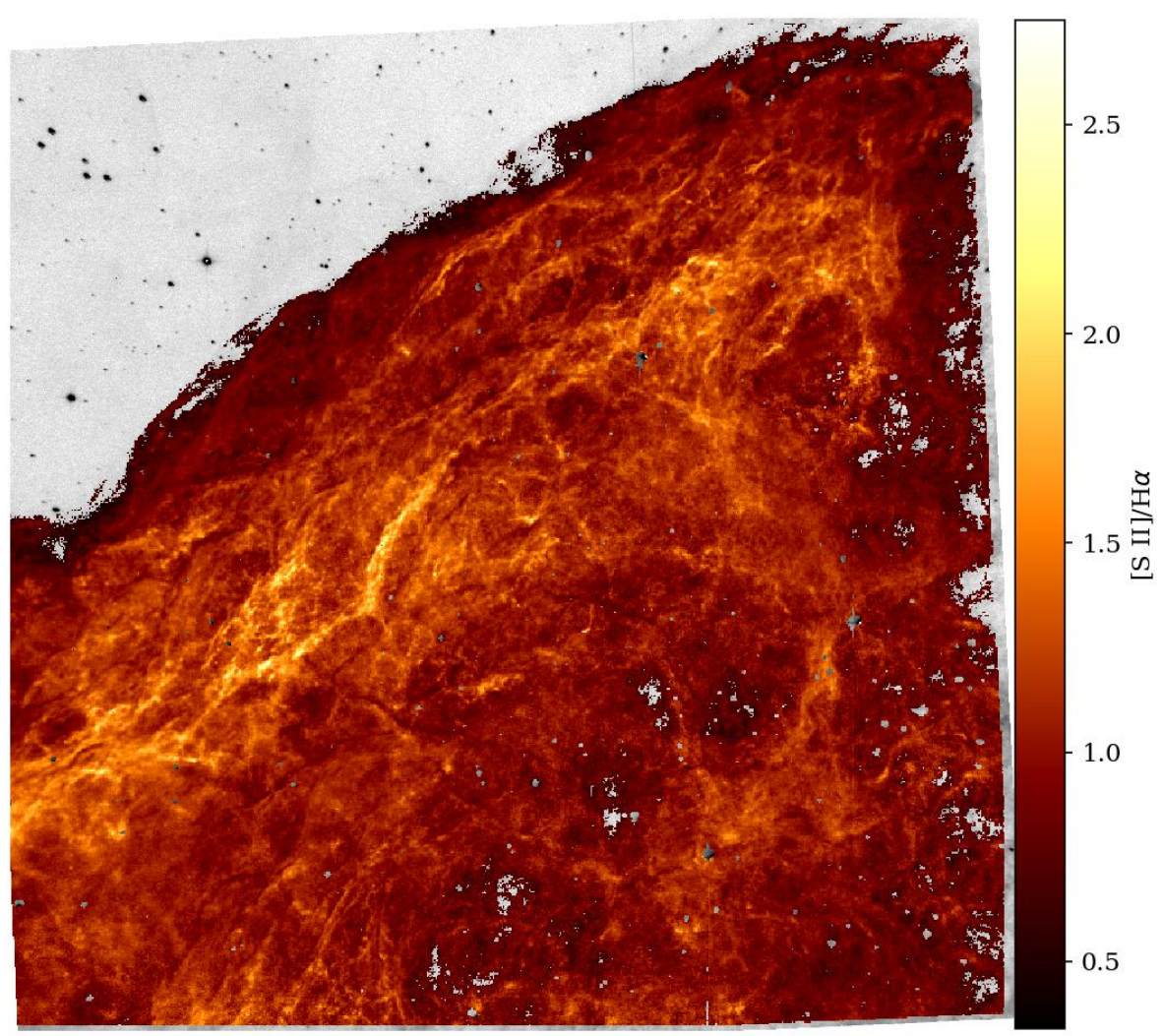

Figure A11. Same as Fig. A7, for $[\mathrm{S} I \mathrm{II}] \lambda 6716+\lambda 6731 / \mathrm{H} \alpha$.

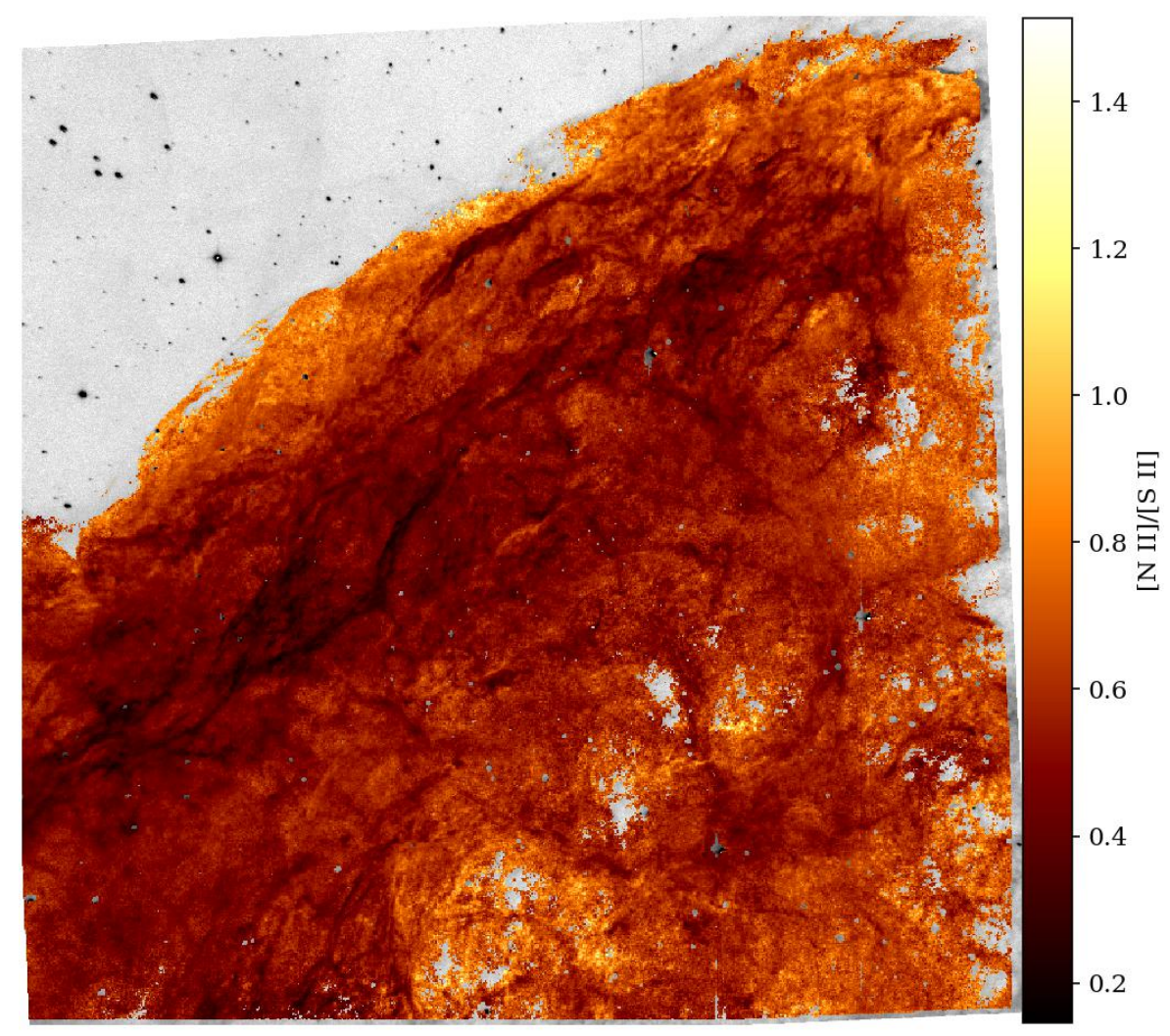

Figure A12. Same as Fig. A7, for $[\mathrm{N}$ II $] \lambda 6583 /[\mathrm{S} \mathrm{II}] \lambda 6716+\lambda 6731$. 


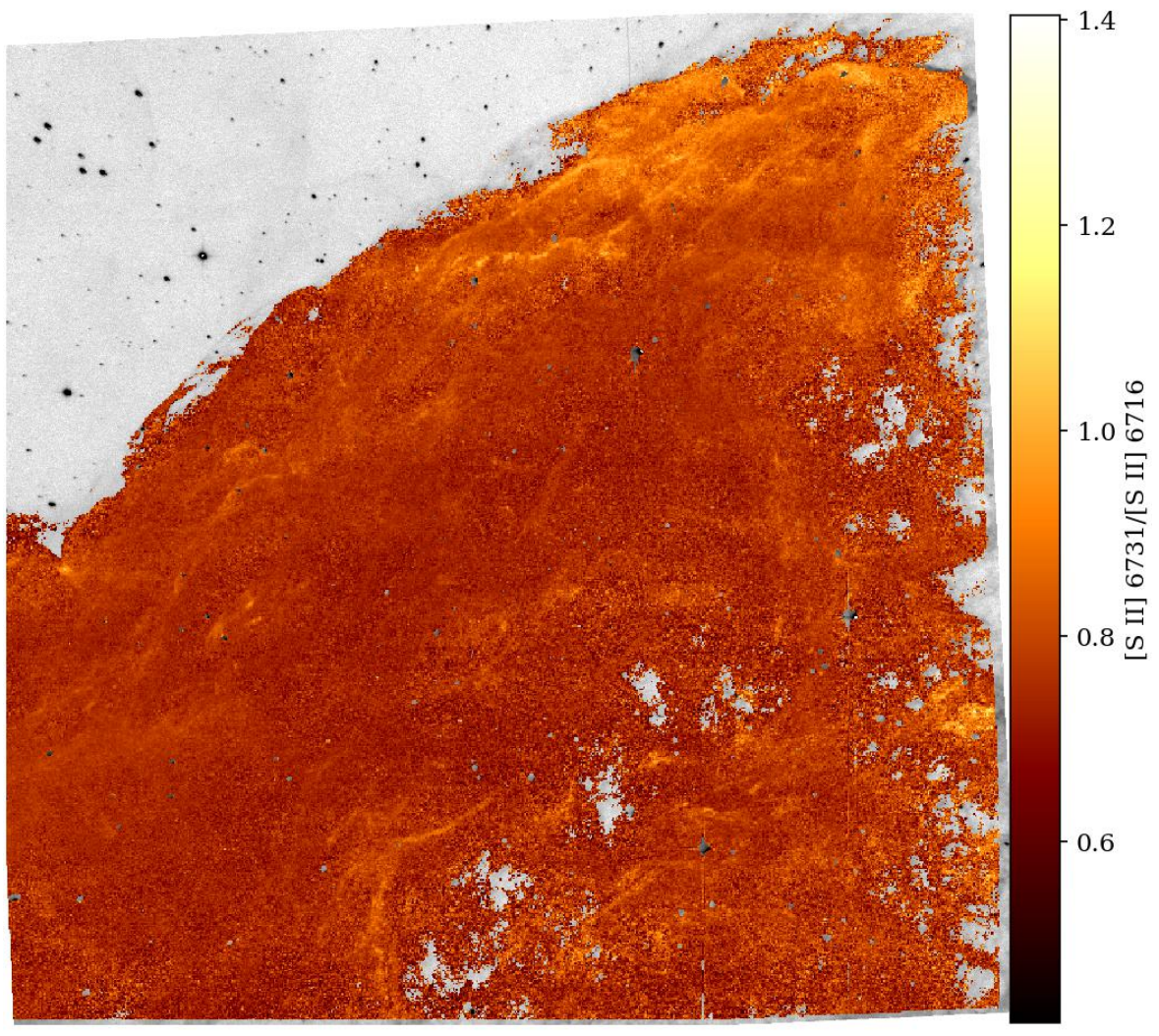

Figure A13. Same as Fig. A7, for [S II] $\lambda 6731 /$ [S II] $\lambda 6716$. 